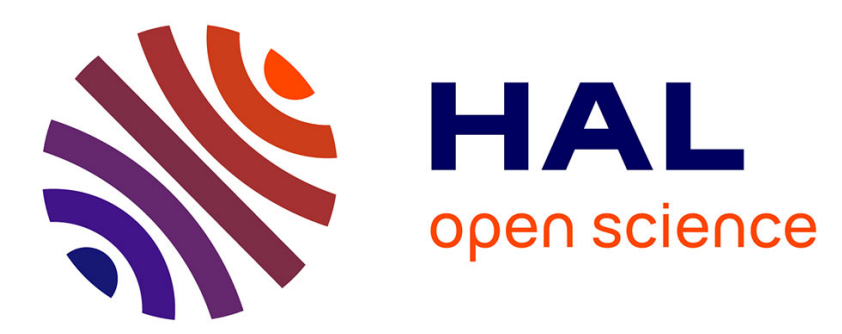

\title{
New Spectrum Efficient Reconfigurable Filtered-OFDM Based L-Band Digital Aeronautical Communication System
}

\author{
Niharika Agrawal, S. Darak, Faouzi Bader
}

\section{> To cite this version:}

Niharika Agrawal, S. Darak, Faouzi Bader. New Spectrum Efficient Reconfigurable Filtered-OFDM

Based L-Band Digital Aeronautical Communication System. IEEE Transactions on Aerospace and Electronic Systems, 2019, 55 (3), pp.1108-1122. 10.1109/taes.2019.2891092 • hal-01984038

\section{HAL Id: hal-01984038 \\ https://hal.science/hal-01984038}

Submitted on 16 Jan 2019

HAL is a multi-disciplinary open access archive for the deposit and dissemination of scientific research documents, whether they are published or not. The documents may come from teaching and research institutions in France or abroad, or from public or private research centers.
L'archive ouverte pluridisciplinaire HAL, est destinée au dépôt et à la diffusion de documents scientifiques de niveau recherche, publiés ou non, émanant des établissements d'enseignement et de recherche français ou étrangers, des laboratoires publics ou privés. 


\title{
New Spectrum Efficient Reconfigurable
}

\section{Filtered-OFDM Based L-Band Digital Aeronautical}

\section{Communication System}

\author{
Niharika Agrawal, Student Member, IEEE,, S. J. Darak, Member, IEEE and Faouzi Bader, Member, IEEE
}

\begin{abstract}
To meet an ever-increasing demand of the spectrum for aeronautical communication, we propose new L-band digital aeronautical communication system (LDACS) using reconfigurable filtered OFDM (Ref-OFDM). The proposed protocol enables transceivers to dynamically adapt the transmission bandwidth over a wide range to meet the desired quality of service, and high out-of-band attenuation leads to significant improvement in the vacant spectrum utilization. We support our claims via simulation and complexity comparison results for various realistic channel conditions.
\end{abstract}

\section{Keywords}

Air to ground communication, L-band Digital Aeronautical Communication System (LDACS), air-traffic management (ATM), Spectrum efficiency, Reconfigurable Filtered OFDM

\section{INTRODUCTION}

The air traffic management (ATM) system handles the communication between the aircraft and between aircraft and ground terminals in the controlled airspace. Existing ATM system can support limited data rate services and fewer communication links due to limited bandwidth. To meet the spectrum demand of ever-increasing air traffic and enable a variety of services to support different phases of flight, international civil aviation organization (ICAO) proposed future communication infrastructure (FCI) system [35, 37, 41]. It consists of several communication data links between satellite stations, aircraft and ground terminals as shown in Fig. 1. Such FCI system is expected to be deployed for other communications, navigation, and surveillance (CNS) applications as well. Ongoing research projects such as the Next Generation Air Transportation System (NextGen) and Single European Sky ATM Research (SESAR) [37] aim to develop and realize such FCI system. The work presented in this paper focuses on the air-to-ground communication (A2GC) which is one of the important data link of the FCI system.

The brief history of the evolution of the A2GC system is depicted in Figure 2. In 1940, the first A2GC link was deployed using the analog modulation based communication system. To improve the robustness and throughout, A2GC link was digitized

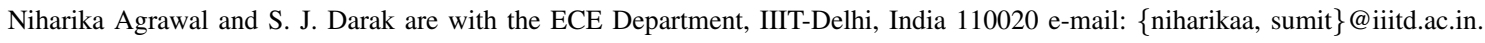

Faouzi Bader is with SCEE/IETR - CentraleSupelec, Rennes, France e-mail: faouzi.bader@supelec.fr. 


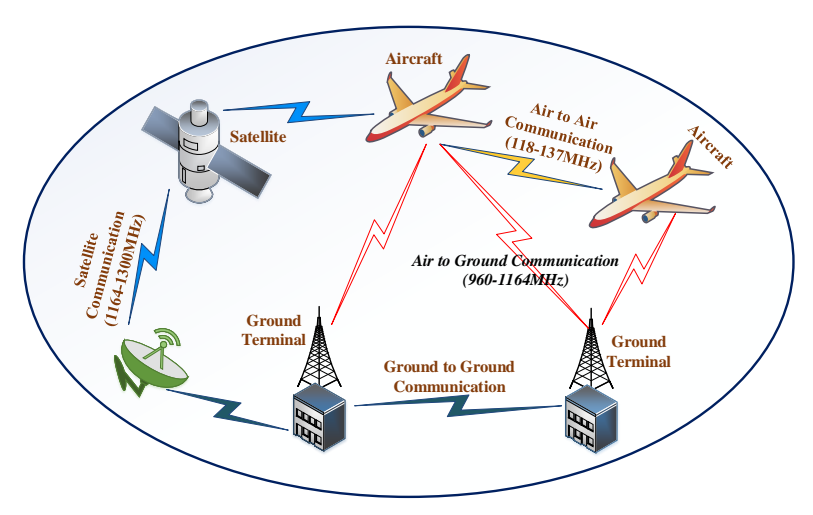

Fig. 1: Various communication links in the future communication infrastructure (FCI) system.

in the 1990's and deployed in the $19 \mathrm{MHz}$ VHF band (118-137 MHz). Hence, it is referred to as VHF Data Link (VDL)[37] and is widely used in existing systems. The VHF band is also used for air-to-air communications as shown in Fig. 1. The drawbacks of the VDL based system are severe congestion due to fewer communication links and low data rate which limits the number of offered services. In 2007, opportunistic spectrum access based inlay approach was identified to enable A2GC in the $L$-band (960-1164MHz), and it is referred to as $L$-band digital aeronautical communication system (LDACS). It is expected to support a wide variety of services ranging from voice, data to futuristic delay-sensitive multimedia services by exploiting vacant frequency bands between incumbent $L$-band users. In 2009, LDACS specifications were finalized, and the first prototype was recently demonstrated $[15,33,37,41]$.

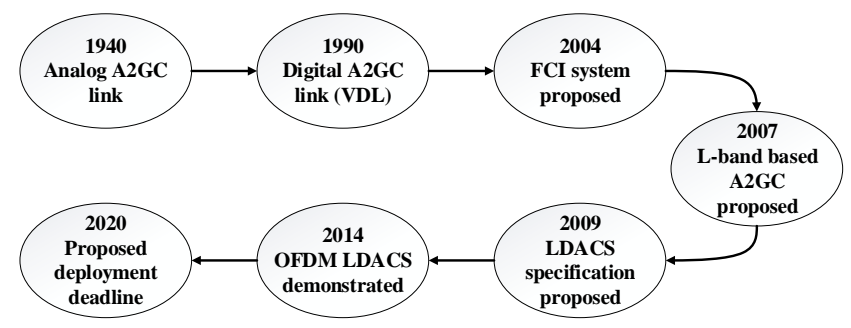

Fig. 2: Brief history about the evolution of A2GC system.

The envisioned LDACS consists of two sub-systems: 1) LDACS1: a Broadband multi-carrier system based on orthogonal frequency division multiplexing (OFDM). It is similar to IEEE 802.16 standard and employs inlay approach between incumbent distance measuring equipment (DME) signals, and 2) LDACS2: Narrowband single carrier system based on time division duplex approach. It is similar to the global system for mobile communication (GSM) and uses a Gaussian minimum shift keying modulation scheme [25]. For the next-generation A2GC system, LDACS1 seems to be a better choice due to the capability to support high-speed delay-sensitive multimedia services and compatibility with the cellular communication standards [29, 33]. Hence, the work presented in this paper will focus on LDACS1, and we will refer to it as LDACS hereafter.

The spectrum occupancy of $L$-band is shown in Figure 3. Various legacy or incumbent users in $L$-band are DME signals (960$1215 \mathrm{MHz}$ ), radar-based multi-functional information distribution system (MIDS), universal access transceiver (UAT) systems 
(978 MHz), secondary surveillance radar (SSR) (1030 MHz) and airborne collision avoidance system (ACAS) (1090 MHz) [15]. The LDACS system can be deployed in the $L$ band using two approaches: overlay and inlay approach. In the overlay approach, it is deployed in the vacant spectrum where no other legacy system is present. This approach is straightforward and chosen for GSM like LDACS2 in 960-975 MHz band. On the other hand, the overlay approach is not suitable for OFDM based LDACS (or LDACS1) due to limited vacant spectrum in the $L$-band. Hence, an inlay approach is envisioned exploiting the multiple 1 $\mathrm{MHz}$ frequency bands between DME signals.

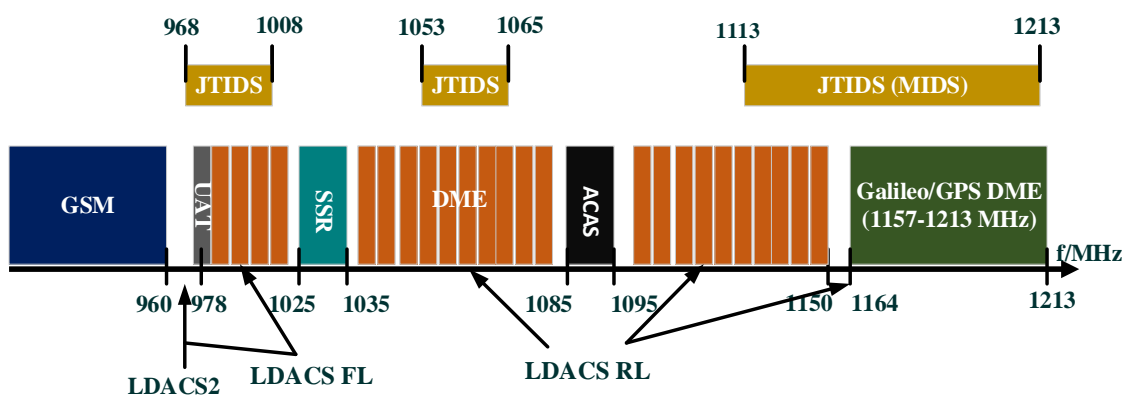

Fig. 3: $L$-band spectrum occupancy and incumbent users.

Existing OFDM-based LDACS protocol can utilize only $498 \mathrm{KHz}$ bandwidth though $1 \mathrm{MHz}$ vacant spectrum is available between adjacent DME signals. The main reason behind less than $50 \%$ of vacant spectrum utilization is the high out-of-band emission due to inherent rectangular filtering in OFDM. Fixed transmission bandwidth also limits the number of supported services and hence, the usefulness of LDACS in other CNS applications. The proposed reconfigurable filtered-OFDM based LDACS enable transceivers to dynamically adapt the transmission bandwidth over a wide range to meet the desired quality of service and high out-of-band attenuation due to filtering allows wider transmission bandwidth as well as multiple narrowband transmissions leading to significant improvement in the vacant spectrum utilization. The contributions of this paper are:

1) The frame structure of the proposed LDACS allows transceivers to adapt the transmission bandwidth from $186 \mathrm{KHz}$ to 732 KHz. It is a generalized version of existing LDACS which supports only $498 \mathrm{KHz}$ bandwidth.

2) We propose a reconfigurable filtered OFDM (Ref-OFDM) using a reconfigurable linear phase multi-band finite impulse response (FIR) digital filter. It can adapt the bandwidth on-the-fly without the need of changing the filter coefficients and can be easily extended to multi-band filter for simultaneous transmission in multiple bands.

3) We theoretically analyze the bit-error-rate (BER) performance of the Ref-OFDM in presence of the DME interference and LDACS wireless channels.

4) Simulation results compare the proposed Ref-OFDM with other waveforms for LDACS specifications. Other waveforms are filtered OFDM (F-OFDM) [11], generalized frequency division multiplexing (GFDM) [28] and filter bank multi-carrier (FBMC) [43].

5) The detailed comparison is done in terms of various parameters such as out-of-band (OOB) emission, interference to incumbent users for different transmission bandwidths, BER for LDACS specific wireless channels.

6) We present computational complexity comparison results which show that the complexity of Ref-OFDM is lower than other 
waveforms except OFDM making the proposed work an attractive solution for next-generation LDACS.

The proposed work is the extended version of our conference paper [3]. Here, we present a new frame structure, improved filter design, and theoretical BER analysis. Also, the study of [3] does not consider LDACS specifications and corresponding channels. In addition to that, we consider multi-user multi-band deployment which has not been discussed yet in the literature. Please refer to [1] for the detailed technical report of this work.

The rest of the paper is organized as follows. The literature review is presented in Section II. Section III and IV show the proposed reconfigurable LDACS protocol and Ref-OFDM waveform design, respectively. The simulation results are presented in Section VI. Section VII concludes the paper.

\section{Literature REVIEW}

The OFDM based LDACS specifications and its usefulness for CNS applications are summarized in [7, 21, 33]. In this section, we review various works which analyze the performance of existing LDACS and contribute to improving its feasibility, robustness, and complexity.

In [12], a new model has been proposed to evaluate and compare the performance of various FCI links as per the Required Communication Performance (RCP) metric introduced by the ICAO. Based on the analysis, they have also identified the desired characteristics for any data link to meet RCP requirements. In [14], the effect of the DME power and pulse rate on the BER of OFDM based LDACS is analyzed. The theoretical results confirm the feasibility of the inlay approach in $L$-band but simulation and experimental results are not presented. Also, the analysis is limited to the DME interference specific to European aerospace. In $[7,10,16,34]$, interference analysis is done via characterizing various incumbent users in $L$-band concerning their spectral characteristics, transmit power and the duty cycle. The simulation results show slight degradation in the BER performance of LDACS when DME power is high. Two algorithms have been proposed to mitigate the DME interference, in [10, 24]. In [10], pulse blanking technique has been proposed for interference mitigation and it is now an integral part of the LDACS. Supervised learning-based DME multipath mitigation technique, its performance, and sensitivity analysis are presented in [24]. The works in $[18,36]$ validate the performance of the fixed-point implementation of LDACS in the presence of incumbent $L$-band users on the hardware testbed. Experiments in real radio environments are being carried out to analyze the effect of a non-linear power amplifier and analog-front-end on the performance of LDACS.

Recently, few works dealing with the design and implementation of OFDM-based LDACS transceiver have been proposed $[5,13,30,31,38,39]$. In [30], novel correlation based synchronization approach for large carrier frequency offsets has been introduced, and its implementation on field programmable gate arrays (FPGA) has shown to consume lower area and power without compromising on the BER performance [31]. In [38], the design of LDACS transceiver via partial reconfiguration approach of the FPGA has been proposed. It offers significant improvement in the power consumption of the transceiver without compromising on the performance. In [39], a novel sensing method for detecting the active LDACS transmissions via multiplierless correlation-based method has been proposed. Results show that the proposed approach offers improved performance especially at low signal-to-noise ratio (SNR) and consumes lesser power than the existing ones.

On the receiver side, reconfigurable low complexity filter and filter bank architectures for channelization and spectrum sensing 
applications have been proposed in [5, 13]. Such architectures are based on frequency response masking approach, and they allow the LDACS receiver to receive and sense single as well as multiple frequency bands simultaneously. In [19], we proposed the implementation of LDACS transceiver on Zynq FPGA platform consisting of Advanced RISC Machine (ARM) processor and FPGA on a single chip. Various configurations of the architecture are realized by dividing it into two sections, one for the ARM and other for FPGA. We demonstrated the flexibility offered by co-design approach to decide which part of the transceiver to implement on FPGA and which on the ARM to meet the given area, delay, and power constraints.

Most of the existing works [5, 10, 30, 31, 38, 39] deal with the improving the performance of the OFDM based LDACS. Rectangular windowing in the OFDM leads to the higher OOB emission and hence, poor spectrum utilization. To design FCI system for various CNS application, it must offer large transmission capacity, low latency, and high elasticity, along with the capability to support a wide variety of services which demands distinct transmission bandwidths. To enable this, transceivers with various other waveforms are explored [3, 23, 26]. In [23], the FBMC waveform has been proposed as an alternative to OFDM in LDACS. The FBMC offers higher vacant spectrum utilization than OFDM due to sub-carrier filtering approach but the work in [23] assumes fixed transmission bandwidth compared to the proposed work where the bandwidth is adaptable. From the architecture perspective, the complexity of FBMC is high, and receiver design is challenging due to complex synchronization and channel equalization techniques. Since the architecture of FBMC is significantly different from that of OFDM, the single transceiver cannot support both waveforms unless they are stacked in parallel. From the future perspective, FBMC cannot be easily extended to multiple antenna configurations which are now a default configuration offering high data rates and improved performance in the deep fading environment. Hence, ICAO expects further research on various windowing/filtering techniques to improve the performance and adaptability of OFDM based LDACS.

\section{Proposed Reconfigurable LDACS Protocol: Frame Structure}

In this section, we present the frame structure of the proposed reconfigurable LDACS protocol that supports multiple transmission bandwidths. The requirements of the frame structure are:

- It should be identical to the existing LDACS frame structure when the transmission bandwidth is $498 \mathrm{KHz}$.

- The sub-carrier spacing should be fixed and equal to $9.76 \mathrm{KHz}$ as it depends on the LDACS deployment environment and hence, can not be chosen arbitrarily.

- The number of symbols per frame should be fixed and independent of the transmission bandwidth.

- It is preferable to use identical synchronization and pilot symbol patterns as in existing LDACS protocol.

Based on an experimental study of channel conditions between aircraft and ground terminals at different phases of the flight, the sub-carrier bandwidth is limited to $9.76 \mathrm{KHz}$, and hence, the symbol duration is $120 \mu \mathrm{s}$ [7, 21, 33]. For these specifications and to support different bandwidths ranging from $100 \mathrm{KHz}$ to $1 \mathrm{MHz}$, the FFT size in the proposed protocol is chosen as 128 compared to 64 in the existing protocol. The proposed frame structure for the revised LDACS protocol depicting the data, pilot, and synchronization symbol patterns and their locations for $732 \mathrm{KHz}$ transmission bandwidth is shown in Figure 4.

The frame consists of 128 sub-carriers with the middle sub-carrier being the DC null sub-carrier. The first two symbols of each sub-carrier are reserved for synchronization. The frame comprises of at the most seven different pilot patterns which are critical 


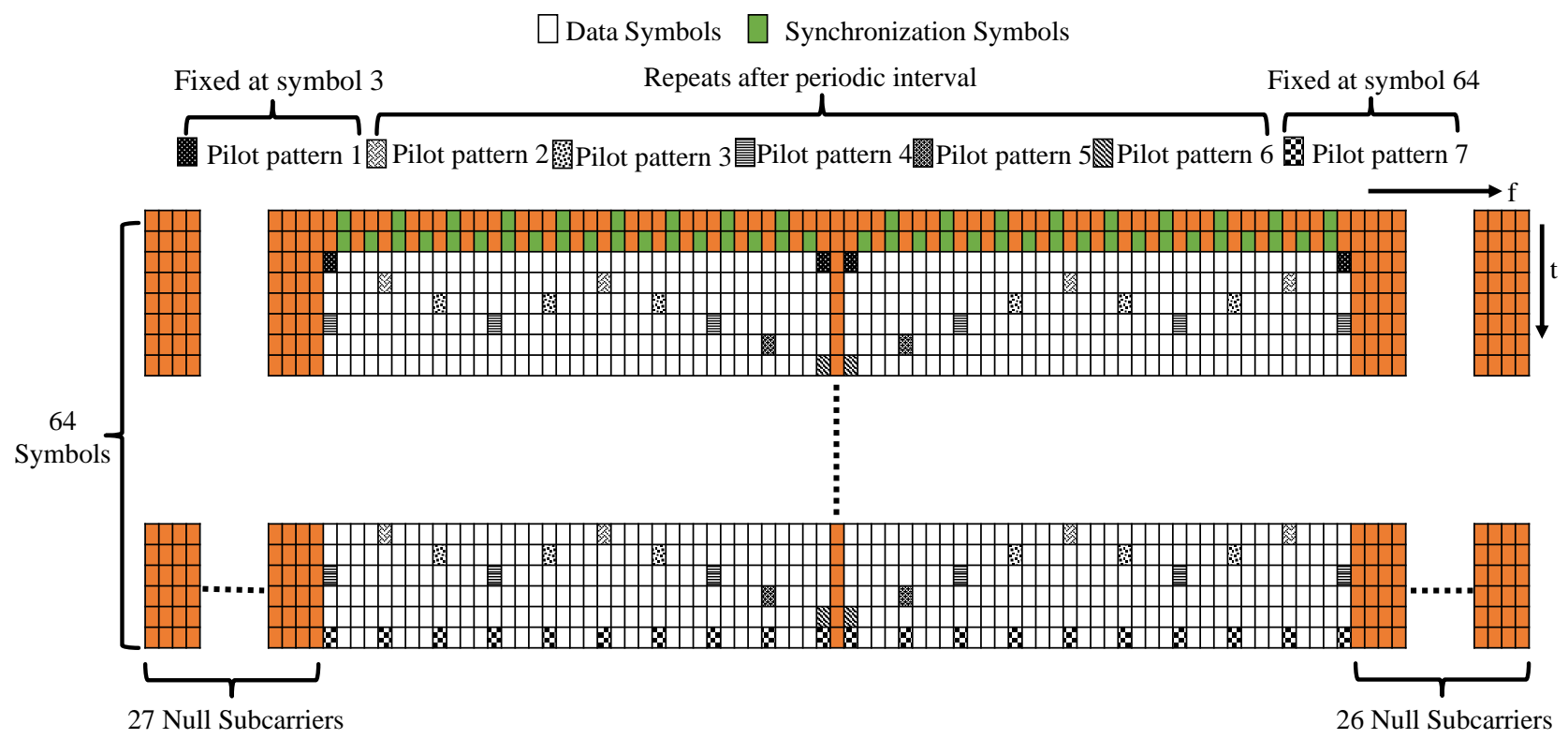

Fig. 4: Proposed frame structure for the revised LDACS protocol depicting the data, pilot and synchronization symbol patterns and locations for $732 \mathrm{KHz}$ transmission bandwidth.

for accurate channel estimation and equalization at the receiver. Similar to the existing protocol, we use fixed pilot patterns, pattern $1(P 1)$ and pattern $7(P 7)$, at the third and last symbols, respectively. Since four sub-carriers separate the pilot symbols in $P 7$ on either side of the DC sub-carrier and the frequency resolution between adjacent sub-carrier is $9.76 \mathrm{KHz}$, the bandwidth can be incremented by $78 \mathrm{KHz}$ only. Based on empirical observations, the bandwidth above $732 \mathrm{KHz}$ is not feasible due to high interference to the DME signal. Also, the bandwidth below $186 \mathrm{KHz}$ may not be suitable for the multi-carrier waveform. Thus, the proposed frame structure supports eight discrete bandwidths which are $732 \mathrm{KHz}, 654 \mathrm{KHz}, 576 \mathrm{KHz}, 498 \mathrm{KHz}, 420 \mathrm{KHz}$, $342 \mathrm{KHz}, 264 \mathrm{KHz}$, and $186 \mathrm{KHz}$. For these bandwidths, the number of symbols should be fixed and multiple of the number of repeating pilot patterns, $P 2-P 6$. For instance, for $732 \mathrm{KHz}-342 \mathrm{KHz}$, all five patterns $(P 2-P 6)$ are used while for the $264 \mathrm{KHz}$ and $186 \mathrm{KHz}$ bandwidth, the patterns used are $P 2-P 5$ and $P 2-P 4$, respectively. Hence, the number of symbols per frame is fixed to 64 out of which 2 are synchronization symbols, and 2 are pilot patterns, $P 1$, and $P 7$. The number of null-sub-carriers on each side depends on the transmission bandwidth as shown in Figure 5.

\section{Proposed Reconfigurable LDACS Protocol: Ref-OFDM WaVeform Based Transceiver}

In this section, we present the design of the proposed Ref-OFDM waveform based transceiver for the proposed frame structure as discussed in the previous section. The motivation behind the proposed transceiver is to support various services that demand distinct bandwidths. For example, consider the scenario in Figure 6 (a) where the existing LDACS can support at the most 498 $\mathrm{KHz}$ bandwidth due to the high out-of-band emission of OFDM. Similarly, it does not allow the multiple users to share the frequency band especially when transmitting over a narrow bandwidth as shown in Figure 6 (b).

The proposed waveform aims to overcome these drawbacks. The requirements of the new waveform are: 


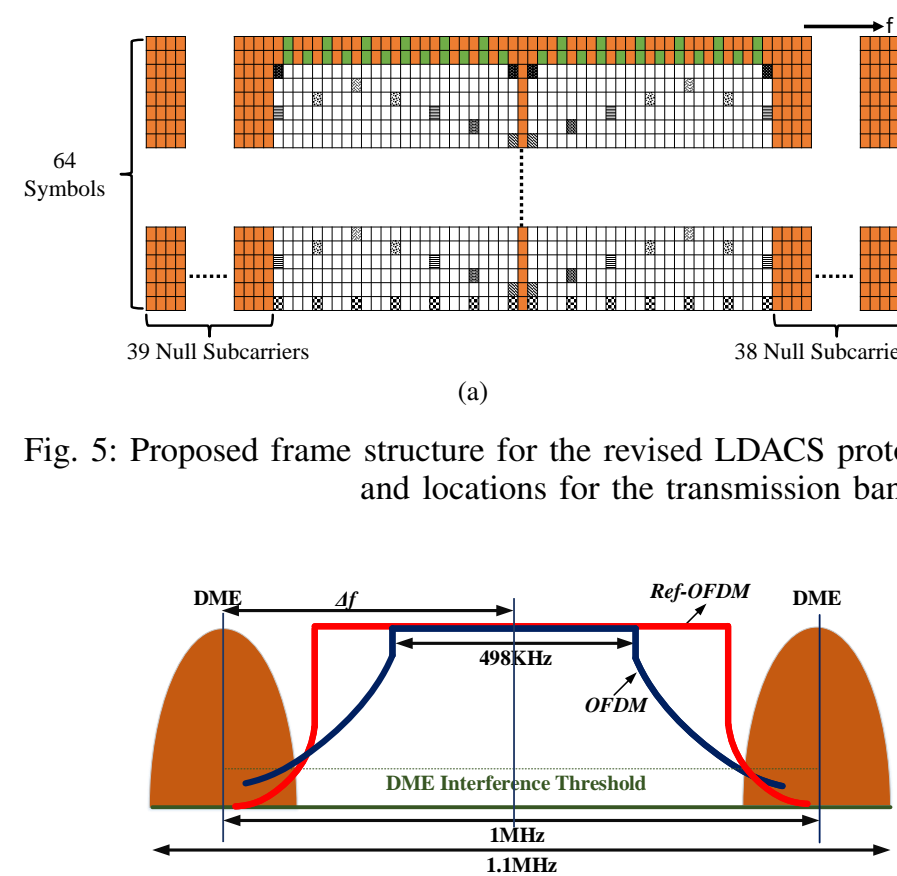

(a)

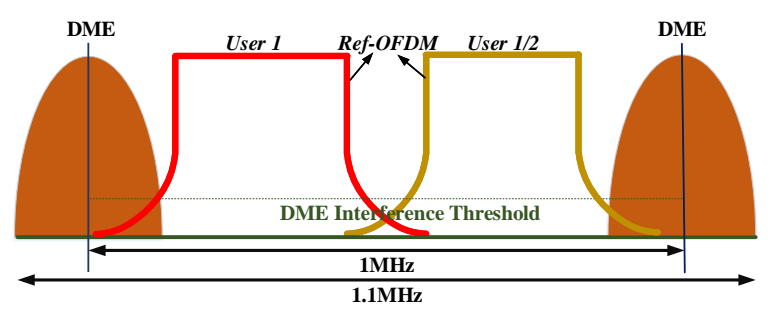

(b)

Fig. 6: (a) Single user, and (b) Multi-user LDACS deployment scenarios for a given DME interference threshold.

- It should allow transceivers to adapt the transmission bandwidth over a wide range without compromising on the interference constraints of the incumbent $L$-band users (e.g., DME).

- The implementation complexity of the transmitter and receiver should be as low as possible.

- It should be compatible with existing OFDM based LDACS transceivers. Ideally, transceivers should be capable of dynamically switching between existing and new waveform on-the-fly.

To begin with, we present detail design of the transmitter followed by the receiver.

\section{A. Ref-OFDM Transmitter}

The block diagram of the Ref-OFDM transmitter is shown in Figure 7. As per the LDACS specifications, it consists of randomizer block which randomizes the input data to be transmitted by XORing with the LDACS randomizer stream. The data is then encoded via Reed Solomen (RS) and Convolutional (CC) encoder with the coding rate as 0.9 and 0.5 , respectively. A helical block interleaver is used to minimize the probability of burst errors. The output of the interleaver is modulated by an appropriate modulation scheme such as QPSK, 16 QAM, and 64 QAM followed by the symbol to frame mapping for given transmission bandwidth. After conventional $N$ point Inverse Fast Fourier Transform (IFFT) and Cyclic Prefix (CP) addition, the discrete time domain signal corresponding to the $k^{\text {th }}$ sub-carrier can be given as,

$$
x[n]=\frac{1}{K} \sum_{k=0}^{K-1} X_{k} e^{\frac{j 2 \pi k n}{K}}
$$


where, $K$ is the IFFT size, $n$ is the discrete time index and $X_{k}$ is frequency domain response of the transmitted signal at the $k^{\text {th }}$ sub-carrier. It is given by

$$
X_{k}=\sum_{n=0}^{K-1} x[n] e^{\frac{-j 2 \pi k n}{K}}
$$

The $x[n]$ is then filtered using the proposed reconfigurable digital filter $f[n]$. Hence, the proposed waveform is referred to as Ref-OFDM waveform. The output of the filter $x^{\prime}[n]$ is appropriately up-sampled and transmitted over the channel via analog front-end and antenna. The transmitted signal $x^{\prime}[n]$ is the convolution $(*)$ of $x[n]$ and the filter $f[n]$ and can be expressed as,

$$
x^{\prime}[n]=f[n] * x[n]
$$

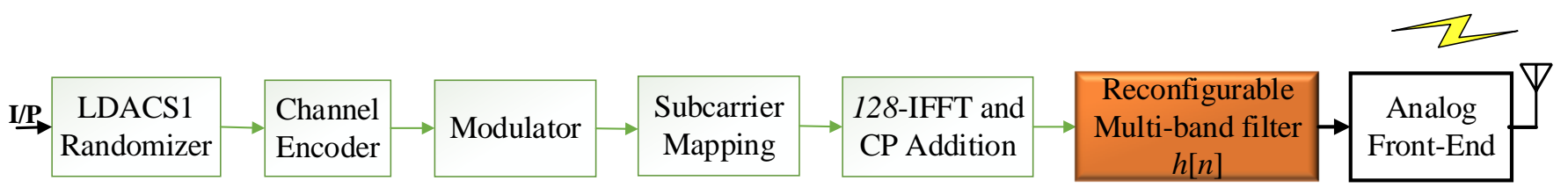

Fig. 7: Block diagram of the REF-OFDM based LDACS transmitter.

Next, we present the design details of the reconfigurable filter.

1) Filter Design for Single Band Transmission: For the proposed LDACS specifications discussed in the previous Section, we need a filter which can support eight different bandwidths. One way to design such a filter is the Velcro approach where eight distinct filters are stacked in parallel [42]. Such approach incurs vast area and power complexity and still offers limited flexibility. In a programmable filter, filter coefficients corresponding to different frequency responses are stored in the memory and retrieved when required [40]. Though less complicated than Velcro approach, reconfiguration time of the programmable filters is high, and it can not take advantage of the methods which significantly reduce the complexity of fixed coefficient filter by replacing the computationally intensive coefficient multiplication operation with the hardware-friendly shift and add operations.

The proposed reconfigurable fixed coefficient filter offers variable bandwidth baseband bandpass responses and is based on the coefficient decimation method (CDM) and its extensions [6, 27]. To begin with, we discuss CDM using a suitable example. Consider the prototype baseband bandpass filter, $F\left(e^{j \omega_{c}}\right)$, where $2 \omega_{c}$ is the bandwidth of the filter. The prototype filter is designed of length $L_{f}$. The filter coefficients are obtained using Parks-McClellan optimal FIR filter design method and the filter response for $\omega_{c}=0.12 \pi$ is shown in the Figure $8(\mathrm{a})$. Note that all frequency specifications in this sub-section are normalized for half the sampling frequency. The CDM can provide the frequency responses with the bandwidth integral multiple of the original bandwidth, $2 \omega_{c}$ using fixed-coefficient prototype filter. Let us consider this integer factor as $D \in\{1,2,3 .$.$\} . In CDM with factor$ $D$, every $D^{t h}$ coefficient of the prototype filter is kept unchanged and remaining coefficients are truncated to zero [27]. This results in the multi-band frequency response, $F^{c d m}\left(e^{j \omega_{c}}\right)$, which is given as 


$$
\left.F^{c d m}\left(e^{j \omega_{c}}\right)=\frac{1}{D} \sum_{i=0}^{D-1} F\left(e^{j\left(\omega_{c}-\frac{2 \pi i}{D}\right.}\right)\right)
$$

Next, every $D^{\text {th }}$ coefficient of the filter is grouped by removing the zero-valued coefficients to obtain the baseband bandpass response with the bandwidth $2 D \omega_{c}$. For example, the frequency responses obtained using the prototype filter in Figure $8($ a) and the CDM with $D=2$ and $D=6$ are shown in Figure $8(\mathrm{~b})$. Note that, the filter coefficients are fixed and independent of $D$. However, the transition bandwidth and stop-band attenuation deteriorate by factor $D$. One way to overcome this deterioration is to over-design the prototype filter with higher order. An extension of CDM referred to as modified CDM (MCDM) [6], offers decimated bandpass response with large bandwidth using a smaller value of $D$ than that required in the CDM. In MCDM, all coefficients except every $D^{t h}$ coefficient of the prototype filter, $F\left(e^{j \omega_{c}}\right)$ are discarded followed by sign reversal of every alternate retained coefficient. The filter response is then given by

$$
F^{m c d m}\left(e^{j \omega_{c}}\right)=\frac{1}{D} \sum_{i=0}^{D-1} F\left(e^{j\left(\omega_{c}-\frac{\pi(2 i+1)}{D}\right)}\right)
$$

For instance, MCDM with factor $D$ results in a baseband bandstop response and the corresponding bandpass response, with bandwidth $1-D \omega_{c}$, can be obtained by subtracting it from an appropriately delayed version of the input signal. For example, the MCDM with $D=2$, offers the bandpass response with the bandwidth of $0.76 \pi$ as shown in Figure 8(c). It has narrower transition bandwidth and better stopband attenuation than the bandpass response with bandwidth $0.72 \pi$ obtained using the CDM in Figure 8(b).

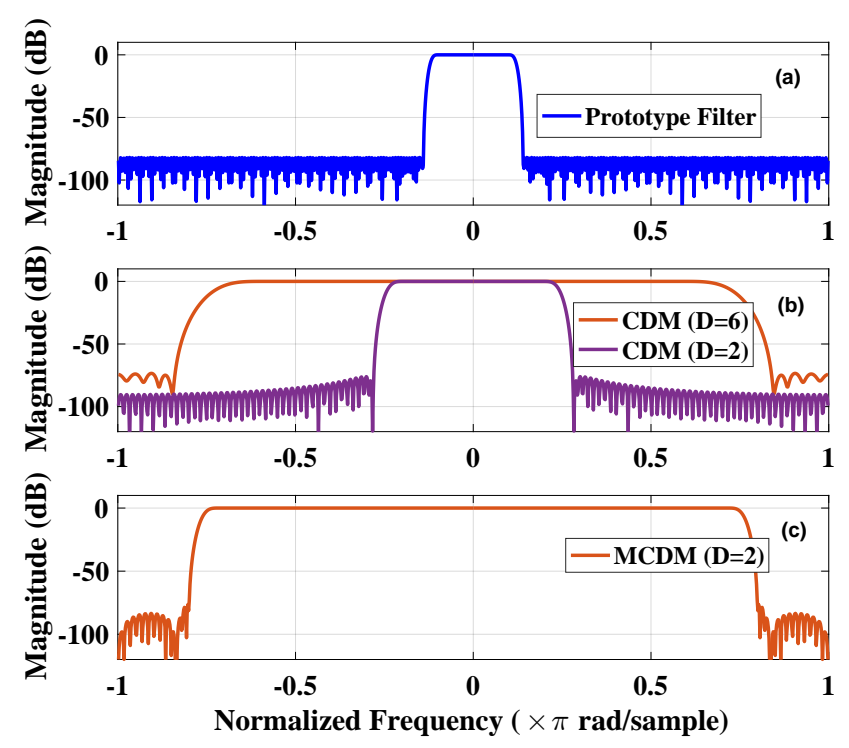

Fig. 8: Reconfigurable filter design using CDM and MCDM. (a) Prototype baseband bandpass filter with $\omega_{c}=0.12 \pi$ (b) Baseband bandpass filter responses with the bandwidth $0.24 \pi$ and $0.72 \pi$ obtained using the CDM approach with $D=2$ and $D=6$, respectively, (c) Baseband bandpass filter responses with the bandwidth $0.76 \pi$ obtained using the MCDM with $D=2$. 
The proposed filter is designed using a combination of CDM and MCDM. For easier understanding, we mention normalized bandwidths corresponding to actual transmission bandwidths in Table I. The maximum input frequency is $1250 \mathrm{MHz}(=128 * 9.76$ $\mathrm{KHz}$ ) which corresponds to the sampling frequency of $2.5 \mathrm{MHz}$. For the desired values of bandwidths, we obtain the bandwidth of the prototype filter as $0.24 \pi$ (i.e., $\omega_{c}=0.12 \pi$ ) and range of $D$ as $\{2,3,4,5,6,7\}$ via dynamic programming. For instance, the MCDM with $D=7$ and prototype filter with $\omega_{c}=0.12 \pi$ give bandpass response with bandwidth $0.32 \pi$ (i.e., $\omega_{c d}=0.16 \pi$ ). Since the CDM and MCDM result in deterioration of the filter response, the prototype filter needs to be over-designed such that the passband ripple, stopband attenuation and transition bandwidth of the prototype filter are $D_{\max }(=7)$ times better than the respective desired values of these parameters. Based on these parameters, order and coefficients of the prototype filter are obtained. For example, for the desired stop-band attenuation, pass-band ripple and transition bandwidth of $70 \mathrm{~dB}, 0.1 \mathrm{~dB}$ and $0.1 \pi$, respectively, the prototype filter order is 240 and bandwidth is $0.24 \pi$, i.e. $\omega_{c}=0.12 \pi$. Please refer to Table I for mapping between the desired bandwidth and corresponding $D$. The baseband bandpass responses for these bandwidths are shown in Figure 9.

TABLE I: Reconfigurable Filter Design

\begin{tabular}{|l|l|l|l|}
\hline $\begin{array}{l}\text { Bandwidth } \\
(\mathrm{KHz})\end{array}$ & $\begin{array}{l}\text { Desired cut-off fre- } \\
\text { quency }\left(\omega_{c d}\right)\end{array}$ & $\begin{array}{l}\text { Decimation Factor } \\
(\mathrm{D})\end{array}$ & Filter Method \\
\hline 186 & 0.16 & 7 & MCDM \\
\hline 264 & 0.22 & 2 & CDM \\
\hline 342 & 0.28 & 6 & MCDM \\
\hline 420 & 0.34 & 3 & CDM \\
\hline 498 & 0.40 & 5 & MCDM \\
\hline 576 & 0.46 & 4 & CDM \\
\hline 654 & 0.52 & 4 & MCDM \\
\hline 732 & 0.58 & 5 & CDM \\
\hline
\end{tabular}

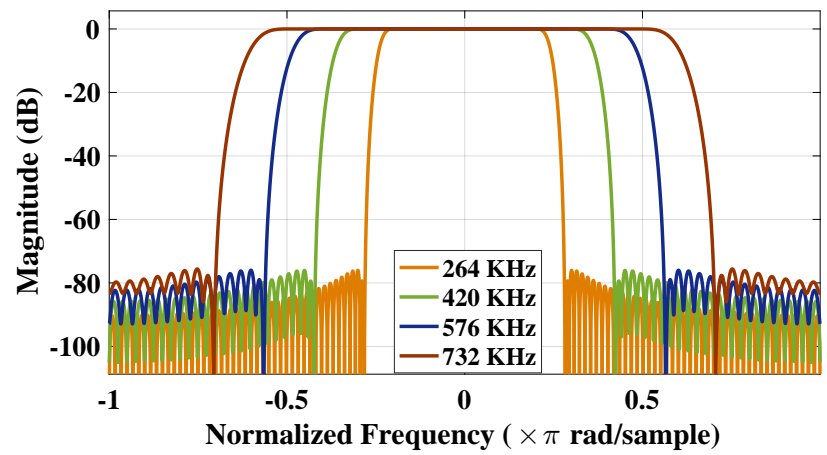

(a)

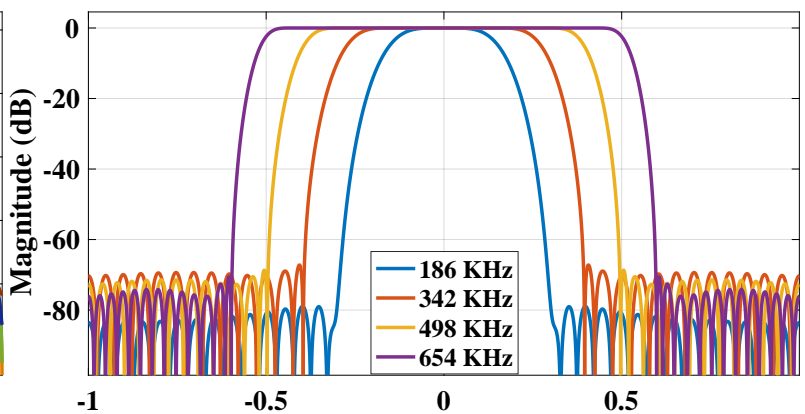

Normalized Frequency $(\times \pi \mathrm{rad} / \mathrm{sample})$

(b)

Fig. 9: Variable baseband bandpass frequency responses obtained using fixed-coefficient baseband bandpass prototype filter with $\omega_{c}=0.12 \pi$ and, (a) CDM , and (b) MCDM approach.

2) Filter Design for Simultaneous Transmission in Multiple Bands: In this subsection, we extend the above reconfigurable filter for the case where user simultaneously transmits in the multiple bands as shown in Figure 6b. It is possible only when the filter provides multi-band frequency response with no image on the other side of the DC frequency. Though CDM offers 
multi-band responses, the response is symmetrical with respect to the DC frequency for real prototype filter. In case of complex prototype filter (i.e. the prototype filter with complex-valued coefficients), the CDM cannot offer variable bandwidth responses for a given center frequency. To obtain an asymmetrical frequency response with variable bandwidth and center frequency, we use conventional modulation based discrete Fourier Transform (DFT) filter bank (DFTFB) approach [20]. In DFTFB, the prototype filter is realized in the polyphase form, and the resultant filter response is modulated using the DFT to obtain bandpass responses at the regular interval between -1 and 1 with no image on the other side of the DC frequency. For example, the DFTFB of order 4 needs 4-point DFT and offers four bandpass responses at the center frequencies of $-1,-0.5,0$ and 0.5 . Note that the bandwidth of all responses is same and equal to the bandwidth of the prototype filter. To obtain the control over the bandwidth, we replace the prototype filter of the DFTFB with the reconfigurable filter discussed in the previous sub-section. Thus, the bandwidth of all the sub-bands is the same and can be tuned to one of the eight supported bandwidths on-the-fly. The control over the center frequency of the bandpass responses can be obtained by choosing the appropriate order of the DFT. For example, the DFTFB of order $K$ offers $K$ bandpass responses located uniformly between -1 and 1 at an interval of $2 / K$ on the normalized frequency scale.

3) Filter Architecture: The architecture of a $K$-band reconfigurable filter is shown in Figure 10. It consists of $N^{\text {th }}$ order prototype filter with real and fixed valued coefficients as $\left\{h_{0}, h_{1}, . . h_{N}\right\}$. It is implemented in the polyphase form with $K$ parallel branches. The sum of the output of all the polyphase branches provides the baseband bandpass response. The output of the polyphase filter is given to the $K$-point DFT as shown in Figure 10 to obtain the multi-band response. Each adder in the conventional FIR filter is replaced with coefficient decimation (CD) block to obtain the bandpass response with variable bandwidth. The CD block either bypass the new coefficient, $h_{C}$ or perform addition operation in case of CDM. In the case of MCDM, CD block needs to perform subtraction operation for alternate retained coefficients. The select signals are used to perform the desired operation on each of the coefficients. The output logic unit is used to obtain the bandpass response by subtracting the bandstop response obtained from the prototype filter and MCDM from the appropriately delayed version of the input signal.

\section{B. Receiver}

The signal at the input of receiver, $r[n]$, consists of three components: 1) The transmitted signal after passed through the LDACS wireless channel with impulse response $h^{L}[n]$, 2) DME interference signal after passed through the LDACS wireless channel with impulse response $h^{D}[n]$, and 3) Zero-mean additive white Gaussian noise $\tilde{n_{0}}[n]$. The $r[n]$ is given as

$$
r[n]=h^{L}[n] * x^{\prime}[n]+h^{D}[n] * s[n]+\tilde{n_{0}}[n]
$$

In LDACS environment, both channels $h^{L}[n]$ and $h^{D}[n]$ assumed to have identical statistics such that

$$
h^{L}[n]=\sum_{l=1}^{L} h_{l}^{L} \delta[n-l] \text { and } h^{D}[n]=\sum_{l=1}^{L} h_{l}^{D} \delta[n-l]
$$

Where $L$ is the total number of channel taps, $h_{l}^{L}$ and $h_{l}^{D}$ are the impulse responses of the channel faced by LDACS and DME 
signal of the $l^{t} h$ path, respectively. The channels are assumed to be time-invariant for each transmitted OFDM symbol.

The receiver performs all the functions similar to the transmitter in the reverse order as shown in the Figure 11.

In the beginning, the received digitized baseband signal is filtered using the same reconfigurable filter $f[n]$ which is the same as the transmitter. The filtered received signal is given by

$$
r^{\prime}[n]=f[n] * h^{L}[n] * f[n] * x[n]+f[n] * h^{D}[n] * s[n]+f[n] * \tilde{n_{0}}[n]
$$

The filtered signal is then passed through the synchronization block to estimate time and frequency offsets. The coarse synchronization is based on correlation of synchronization symbols at the beginning of the forward link frame, and fine synchronization is based on correlation of cyclic prefix of each OFDM symbol. Pulse Blanking technique used to remove the non-linearities and interference. After FFT, channel coefficients and impulse response are estimated using pilots followed by channel equalization via zero forcing approach. In the end, the symbols are demodulated and decoded to obtain the transmitted data. The received signal $R_{k}$ at $k^{t h}$ subcarrier is as follows:

$$
R_{k}^{\prime}=F_{k} H_{k} F_{k} X_{k}+F_{k} H_{d_{k}} S_{k}+F_{k} \tilde{N}_{0} k
$$

where, $H_{k}, H_{d_{k}}$ are the LDACS and DME channel frequency responses at the $k^{t h}$ subcarrier respectively can be given as

$$
H_{k}=\sum_{l=1}^{L} h_{l}^{L} e^{\frac{-j 2 \pi k l}{N}} \text { and } H_{d_{k}}=\sum_{l=1}^{L} h_{l}^{D} e^{\frac{-j 2 \pi k l}{N}}
$$

Next, we obtain the frequency response of the filter $f[n]$ at the $k^{t h}$ subcarrier $F_{k}$. Here, the length of the filter, $f[n]$, is $\left\lceil\frac{\left(L_{f}+1\right)}{D}\right\rceil$ and it is always less than the FFT size, $K$. Thus, the impulse response of the filter is zero padded by $\left(K-\left\lceil\frac{\left.L_{f}+1\right)}{D}\right\rceil\right)$

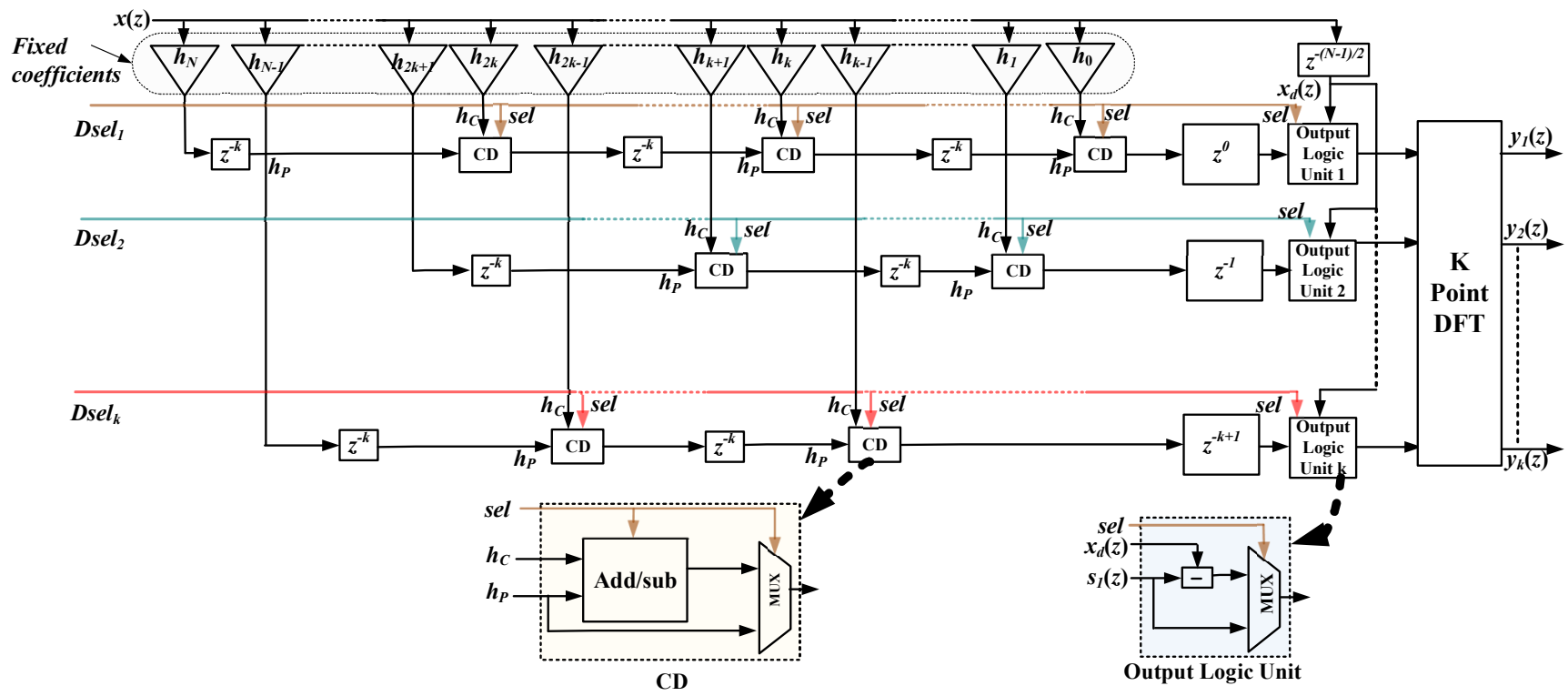

Fig. 10: Architecture of the proposed reconfigurable $K$-band filter. 


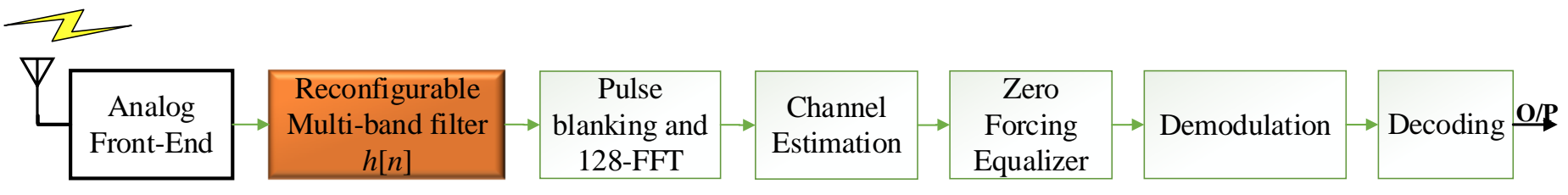

Fig. 11: Block diagram of REF-OFDM based LDACS receiver.

to have the filter length same as FFT length. Then, we obtain $F_{k}$ by taking $K$-point FFT of the zero padded filter impulse response where

$$
F_{k}=W^{H} \cdot\left[f[n], \mathbf{0}_{\left.1 \times\left(K-\left\lceil\frac{\left(L_{f}+1\right)}{D}\right\rceil\right)\right]}\right.
$$

Here, $\mathrm{W}$ is the $K$-point FFT matrix.

In the proposed reconfigurable filter, prototype filter coefficients are fixed, and various responses are obtained using CDM and MCDM approaches. The impulse response of the proposed filter $f[n]$ with CDM factor $D$ is given by

$$
f[n]=f^{P}[n] . b[n]
$$

where, $f^{P}[n]$ be the impulse response of the prototype filter and $b[n]$ can be given as

$$
b[n]=\left\{\begin{array}{cc}
1 & \forall n=m D ; m=0,1,2 \ldots \\
0 & \text { otherwise }
\end{array}\right.
$$

The function $b[n]$ is periodic with period $M$, and hence the Fourier series expansion is given by

$$
b[n]=\frac{1}{D} \sum_{i=0}^{D-1} B(i) e^{\frac{j 2 \pi i n}{D}}
$$

where $B(i)$ are complex-valued Fourier series coefficients defined by,

$$
B(i)=\sum_{n=0}^{D-1} b[n] e^{\frac{-j 2 \pi i n}{D}}
$$

By substituting the Eq. (13) into (15) we will get,

$$
B(i)=\left\{\begin{array}{cc}
1 & \forall k \\
0 & \text { otherwise }
\end{array}\right.
$$

Hence, from Eq. (16) and . (14), $b[n]$ can be expressed as,

$$
b[n]=\frac{1}{D} \sum_{i=0}^{D-1} e^{\frac{j 2 \pi i n}{D}}
$$


By substituting Eq. (17) into Eq. (12), we compute $f[n]$. Similar approach can be used for MCDM. Next, we will analyze the BER performance of the Ref-OFDM based LDACS system for the received filtered signal represented by Eq. (9).

\section{BER Analysis}

The Signal to interference plus noise ratio is the ratio of signal power and the sum of interference and noise power. For the received signal in Eq. (9), the SINR for $k^{t h}$ subcarrier is given as

$$
\operatorname{SINR}(k)=\frac{F_{k}{ }^{4} \lambda_{k} P}{{F_{k}}^{2} P_{\tilde{N}_{0}}+F_{k}{ }^{2} \lambda_{d_{k}} P_{D M E}}
$$

By rewriting the equation (18) we get,

$$
\operatorname{SINR}(k)=\frac{F_{k}^{2} \lambda_{k} P}{P_{\tilde{N}_{0}}+\lambda_{d_{k}} P_{D M E}}
$$

where, $P$ is the transmitted signal power, $F_{k}$ is given by Eq. (11) and $\lambda_{k}=\left|H_{k}\right|^{2}, \lambda_{d_{k}}=\left|H_{d_{k}}\right|^{2}$ are the power associated with the channel frequency responses $\left|H_{k}\right|$ and $\left|H_{d_{k}}\right|$ respectively and can be acquired using (10),

$$
\lambda_{k}=\left|\sum_{l=1}^{L} h_{l}^{L} e^{\frac{-j 2 \pi k l}{N}}\right|^{2} \text { and } \lambda_{d_{k}}=\left|\sum_{l=1}^{L} h_{l}^{D} e^{\frac{-j 2 \pi k l}{N}}\right|^{2}
$$

Here, we are considering that the multipath channel is modelled as the Rayleigh fading channel whose probability density function can be represented as

$$
p_{\lambda}(\lambda)=\frac{1}{\bar{\lambda}} e^{\frac{-\lambda}{\lambda}}
$$

where, $\bar{\lambda}$ is the mean of the variable $\lambda$ and is equal to variance of $H_{k}$. The DME channel $\lambda_{d_{k}}$ also follows the same exponential distribution as it is assumed to have the same distribution as the LDACS channel.

Next, we obtain the expression for DME interference power, $P_{D M E}$. The DME is a transponder-based navigation system used to measure the slant range distance [14]. It is composed of Gaussian shaped pulse pairs. The time and frequency domain representations of the DME signal is given by Eq. (22) and Eq. (23) respectively.

$$
\begin{gathered}
s(t)=e^{\frac{-\alpha t^{2}}{2}}+e^{\frac{-\alpha(t-\Delta t)^{2}}{2}} \\
S(f)=A \sqrt{\frac{8 \pi}{\alpha}} e^{\frac{-2 \pi^{2} f^{2}}{\alpha}} e^{j \pi f \Delta t} \cos (\pi f \Delta t)
\end{gathered}
$$

where, $\alpha$ is pulse width of $4.5 * 10^{-11} s^{-2}, \Delta t$ represents the spacing of the pulses (=12 $\left.\mu s\right)$ and $A$ is constant depending on the power of DME signal. Then, we have

$$
P_{D M E}=\int_{f_{1}}^{f_{2}}|S(f)|^{2} d f
$$

Substituting Eq. (23) in to Eq. (25) and substituting $\cos (\theta)=\frac{e^{j \theta}+e^{-j \theta}}{2}$, we get 


$$
P_{D M E}=A^{2}\left(\frac{8 \pi}{\alpha}\right) \int_{f_{1}}^{f_{2}}\left|e^{\frac{-2 \pi^{2} f^{2}}{\alpha}}\right|^{2}\left|e^{j 2 \pi f \Delta t}\right|^{2} \times\left|\frac{e^{j \pi f \Delta t}+e^{-j \pi f \Delta t}}{2}\right|^{2} d f
$$

Using the Euler formula, $\left|e^{j \theta}\right|^{2}=1$ and assuming, $C_{1}=\frac{4 \pi^{2}}{\alpha}$ and $C_{2}=j 2 \pi \Delta t$, we have

$$
P_{D M E}=A^{2}\left(\frac{8 \pi}{\alpha}\right)\left[2 \int_{f 1}^{f 2} e^{-C_{1} f^{2}} d f+\int_{f 1}^{f 2} e^{-C_{1} f^{2}+C_{2} f} d f+\int_{f 1}^{f 2} e^{-C_{1} f^{2}-C_{2} f} d f\right]
$$

After further algebraic simplification, we get

$$
\begin{aligned}
P_{D M E}= & A^{2}\left(\frac{8 \pi}{\alpha}\right) \sqrt{\frac{\pi}{C_{1}}}\left[\left\{\left(\operatorname{erf}\left(\sqrt{C_{1}} f_{2}\right)-\operatorname{erf}\left(\sqrt{C_{1}} f_{1}\right)\right)\right\}\right. \\
& +\frac{1}{2}\left\{e^{\frac{C_{2}^{2}}{4 C_{1}}}\left(\operatorname{erf}\left(\frac{2 C_{1} f_{2}-C_{2}}{2 \sqrt{C_{1}}}\right)-\operatorname{erf}\left(\frac{2 C_{1} f_{1}-C_{2}}{2 \sqrt{C_{1}}}\right)\right\}\right. \\
& +\frac{1}{2}\left\{e^{\frac{C_{2}^{2}}{4 C_{1}}}\left(\operatorname{erf}\left(\frac{2 C_{1} f_{2}+C_{2}}{2 \sqrt{C_{1}}}\right)-\operatorname{erf}\left(\frac{2 C_{1} f_{1}+C_{2}}{2 \sqrt{C_{1}}}\right)\right\}\right]
\end{aligned}
$$

Using Eq. (19), the BER of the $k^{t h}$ received symbol for M-QAM can be expressed as,

$$
P_{e_{M Q A M}}^{\lambda, \lambda_{d}}(k) \cong \frac{4}{\log _{2} M}\left(1-\frac{1}{\sqrt{M}}\right) \sum_{i=1}^{\sqrt{M} / 2} Q(2 i-1) \times \sqrt{\frac{3 \log _{2} M F_{k}^{2} \lambda_{k} P}{(M-1)\left(P_{\tilde{N}_{0}}+\lambda_{d_{k}} P_{D M E}\right)}}
$$

where erfc(.) is a complex error function [32]. Therefore, BER averaged across the fading channel can be expressed as,

$$
P_{e_{M Q A M}}(k)=E\left[P_{e_{M Q A M}}^{\lambda, \lambda_{d}}(k)\right] \cong \int_{0}^{\infty} \int_{0}^{\infty} P_{e_{M Q A M}}^{\lambda_{1}, \lambda_{2}}(k) \times p_{\lambda}(\lambda) d \lambda p_{\lambda_{d}}\left(\lambda_{d}\right) d \lambda_{d}
$$

The average BER across all the subcarriers is given by

$$
P_{e_{M Q A M}}=\frac{1}{K} \sum_{k=0}^{K-1} P_{e_{M Q A M}}(k)
$$

The analytical expression in Eq. (29) can be evaluated by numerical methods. This completes the BER analysis of the proposed Ref-OFDM based LDACS in presence of the DME interference and multipath Rayleigh fading channel. Next, we present the simulation results to analyze the performance of various LDACS.

Furthermore, the size of the DFT can be changed on-the-fly as per the desired center frequency of the transmission. Such flexibility is not possible in case of OFDM, FBMC and GFDM based LDACS. In addition, Ref-OFDM can be easily extended to a multi-antenna system, unlike FBMC based LDACS. Also, the Ref-OFDM and FBMC waveforms have much better-localized frequency spectrum than OFDM because of the sub-band filtering. Next, we present the simulation results to analyze the performance of various waveforms.

\section{Simulation Results}

In this section, we present extensive simulation results to compare the performance of the proposed Ref-OFDM based LDACS protocol with the existing protocol and FBMC based LDACS in [23] in realistic LDACS environment. Note that we consider the 
revised OFDM based LDACS which employs time domain windowing at the transmitter to improve the out-of-band attenuation. In addition, we also consider the LDACS protocol using GFDM waveform which has not been studied in the literature yet. The results include the comparison of these variants with respect to their out-of-band emission using the power spectral density (PSD) plots for various bandwidths, interference at the adjacent DME signal for these bandwidths, BER in the presence of DME and GGI interference, and implementation complexity. The simulation parameters are chosen as per the LDACS specifications and are given in Table II.

TABLE II: Simulation Parameters

\begin{tabular}{|l|l|}
\hline Parameters & Value \\
\hline Total Bandwidth & $1.25 \mathrm{MHz}$ \\
Transmitted Bandwidth & Any of the supported bandwidths \\
Length of FFT & 128 \\
Used sub-carriers & $18-74$ \\
Sub-carrier spacing & $9.76 \mathrm{KHz}$ \\
Total Symbol duration & $120 \mu \mathrm{s}$ \\
Modulation & QPSK \\
Channel & ENR, APT, TMA \\
CC encoder rate & 0.5 \\
RS encoder rate & 0.9 \\
\hline
\end{tabular}

For LDACS environment, three channels are modeled: Airport (APT), Terminal Manoeuvring Area (TMA), En-routing (ENR). They are modeled as wide sense stationary uncorrelated scattering channels and characterized using three properties: fading, delay paths, and Doppler frequency [17]. The detailed information about the channel models is given in [1].

The channel parameters are given in Table III $[8,9,17,22,33]$. Note that the Doppler frequency is obtained as $F_{D}=F_{c} \times \frac{v}{c}$ where $F_{c}$ is the carrier frequency and is at most $1215 \mathrm{MHz}, v$ is the velocity of the aircraft in $\mathrm{m} / \mathrm{s}$ (1 Knots True Airspeed $(\mathrm{KTAS})=0.5144 \mathrm{~m} / \mathrm{s})$ and $c=299792458 \mathrm{~m} / \mathrm{s}$.

TABLE III: Channel Parameters

\begin{tabular}{|l|l|l|l|l|l|}
\hline Scenario & Fading & Max Delay $(\mu \mathrm{s})$ & $\begin{array}{l}\text { Acceleration } \\
\left(\mathrm{m} / \mathrm{s}^{2}\right)\end{array}$ & Velocity $(\mathrm{KTAS})$ & Doppler Frequency $(\mathrm{Hz})$ \\
\hline APT & Rayleigh & 3 & 5 & 200 & $(1215 e 6) \times \frac{200 \times .5144}{299792458}=413$ \\
\hline TMA & Rician & 20 & 50 & 300 & $(1215 e 6) \times \frac{300 \times .5144}{299792458}=624$ \\
\hline ENR & Rician & 15 & 50 & 600 & $(1215 e 6) \times \frac{600 \times .5144}{299792458}=1250$ \\
\hline
\end{tabular}

\section{A. Single Band Transmission}

To begin with, we consider single user transmitting in the frequency band between adjacent DME signals. For illustration, we consider two bandwidths, 1) $732 \mathrm{KHz}$ which incurs maximum interference to the DME, and 2) $498 \mathrm{KHz}$ which is maximum bandwidth allowed in existing OFDM based LDACS. The corresponding PSD plots for ENR channel are shown in Figure 12. For clarity of the plots, we are only showing the main lobes of the DME signals. It can be observed that the interference at the DME signal is quite high in case of OFDM and GFDM based LDACS (For actual values, please refer to discussion related 
to Table IV at the end of this subsection). The proposed REF-OFDM and FBMC based LDACS can achieve the transmission bandwidths of up to $732 \mathrm{KHz}$ due to high out-of-band attenuation leading to $50 \%$ improvement in spectrum utilization. The PSD plots corresponding to the other two channels are not shown to avoid repetitive plots. However, we have considered them for BER analysis.

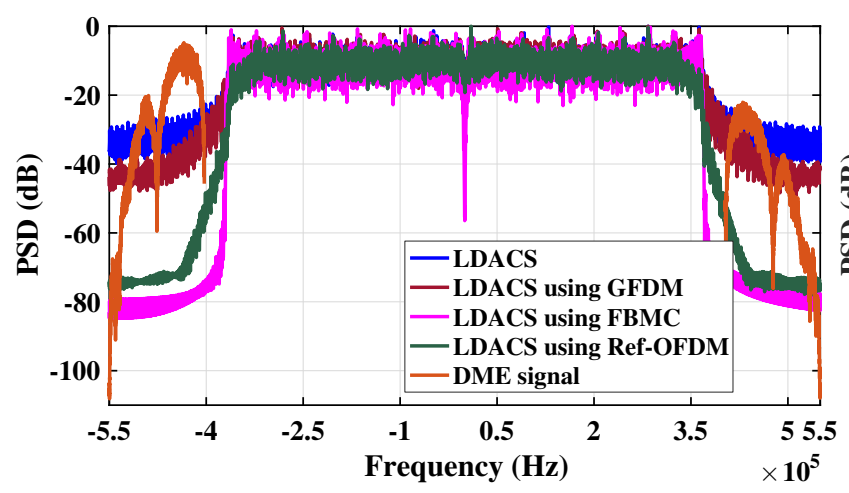

(a)

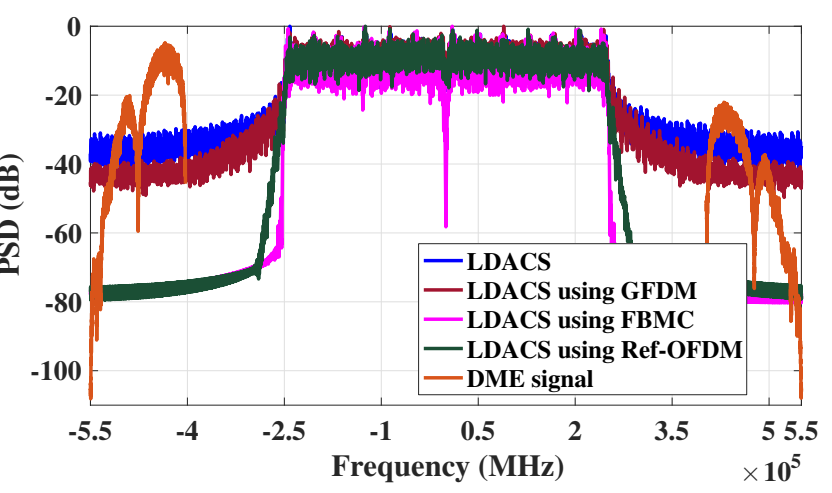

(b)

Fig. 12: The PSD comparison of various waveforms for ENR channel and two different transmission bandwidths, (a) 732KHz, and (b) $498 \mathrm{KHz}$.

Next, we compare the BER of these waveforms for three different channel conditions in the presence of GGI interference only. We do not include BER plots for FBMC as they are overlapping with OFDM BER plots. We again consider $732 \mathrm{KHz}$, and $498 \mathrm{KHz}$ bandwidth and corresponding plots are shown in Fig. 13 (a) and (b) respectively. It can be observed that Ref-OFDM has better BER performance in both the cases. The Fig. 13 (b) shows that for higher transmission bandwidth OFDM and GFDM has very bad ber performance while Ref-OFDM performs much better than other two as there is less DME interference in case of Ref-OFDM. As expected, the performance is better in case of ENR channel due to strong LOS path. The PSD and BER plots show that the Ref-OFDM has better out-of-band attenuation than OFDM and GFDM without compromising on the BER performance.

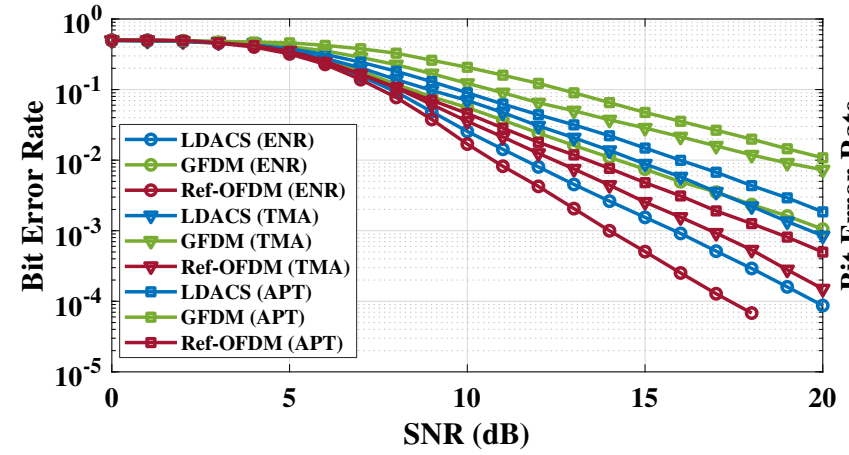

(a)

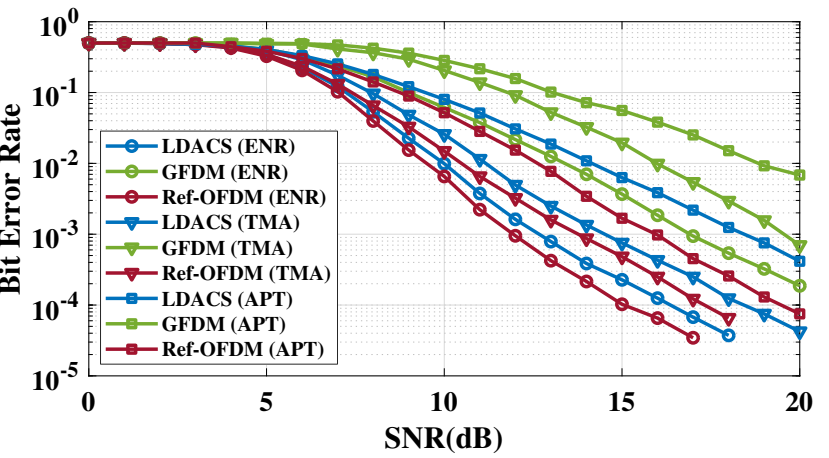

(b)

Fig. 13: The BER comparison of various waveforms for two different transmission bandwidths, (a) $732 \mathrm{KHz}$, and (b) $498 \mathrm{KHz}$ and three different channels. 
Next, we compare the BER of these waveforms in the presence of DME interference for ENR channel. Here, we consider the transmission bandwidth of $342 \mathrm{KHz}$ and three center frequency with a deviation of 0,100 and $400 \mathrm{KHz}$ from the baseband. As shown in Fig. 14, the BER of the proposed Ref-OFDM is significantly better than existing LDACS for all the center frequencies considered here. We also observed that the difference between the BER performance of the Ref-OFDM and OFDM increases with the increase in the transmission bandwidth. The BER of GFDM is worse than that of OFDM while the BER of the FBMC is nearly identical to that of the Ref-OFDM. Similar behavior has also been observed for other channels. These results are not included here for clarity of the plots.

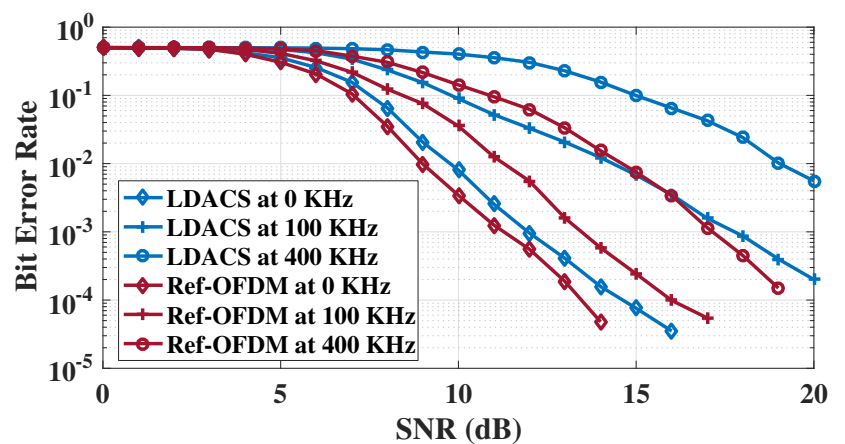

Fig. 14: The BER comparison of Ref-OFDM and OFDM based LDACS in presence of DME interference for ENR channel with $342 \mathrm{KHz}$ transmission bandwidth and three different center frequencies.

Next, we study the interference at the DME signals due to LDACS transmission with the transmission bandwidth of $732 \mathrm{KHz}$ and $498 \mathrm{KHz}$. The interference $(I)$ is calculated by the sum of PSD $(\phi(f))$ between two frequencies $\left(f_{1}\right.$ and $\left.f_{2}\right)$ and can be represented by:

$$
I=\int_{f_{1}}^{f_{2}} \phi(f) d f
$$

Here, we consider LDACS signal at different center frequencies located at an interval of $50 \mathrm{KHz}$ with the DME signal located at the fixed center frequency. The corresponding interference values are shown in Table IV where NA refers to not applicable since corresponding center frequencies are not allowed for the requested bandwidth due to overlap with the main lobe of the DME signals. It can be observed that the proposed Ref-OFDM and FBMC based LDACS offer the lowest interference to the incumbent DME signals. In most of the cases, the interference is lower than $40 \mathrm{~dB}$ which is the desired threshold as per the LDACS requirement and approximately $35 \mathrm{~dB}$ better than existing LDACS. These results not only validate the superiority of the proposed waveform but also indicates the feasibility of multi-band multi-user deployment in case of Ref-OFDM and FBMC based LDACS.

\section{B. Multi-band Transmission}

Here, we consider one user is transmitting in two non-contiguous bands of $186 \mathrm{KHz}$ bandwidth with baseband center frequencies of $-200 \mathrm{KHz}$ and $200 \mathrm{KHz}$. It can be observed from the PSD plots in Figure 15 that the performance of OFDM and GFDM based LDACS have degraded further compared to single band transmission. For instance, the interference at DME signal for 
TABLE IV: Interference at DME in dB Due to Various Waveforms for Transmission Bandwidths of $498 \mathrm{KHz}$ and $732 \mathrm{KHz}$

\begin{tabular}{|c|c|c|c|c|c|c|}
\hline \multirow{2}{*}{ Bandwidth (BW) } & \multirow{2}{*}{ Waveform } & \multicolumn{4}{|c|}{ Transmission Center frequency w.r.t the DME center frequency $(\mathbf{r}=\mathbf{5 0}$ KHz) } \\
\cline { 2 - 7 } & & $\frac{B W}{2}+r$ & $\frac{B W}{2}+2 r$ & $\frac{B W}{2}+3 r$ & $\frac{B W}{2}+4 r$ & $\frac{B W}{2}+5 r$ \\
\hline \multirow{3}{*}{$498 \mathbf{K H z}$} & OFDM & 7.1745 & 2.7038 & 0.9269 & -0.1658 & -0.8701 \\
\cline { 2 - 7 } & GFDM & 5.9571 & -3.1187 & -7.3229 & -8.7974 & -9.3662 \\
\cline { 2 - 7 } & Ref-OFDM & 0.3596 & -38.7566 & -40.7823 & -42.1427 & -43.1139 \\
\cline { 2 - 7 } & FBMC & -2.0064 & -37.8831 & -39.8368 & -40.9379 & -41.6017 \\
\hline \multirow{3}{*}{$732 \mathbf{K H z}$} & OFDM & 6.4676 & 1.8911 & NA & NA & NA \\
\cline { 2 - 7 } & GFDM & 5.2885 & -5.6320 & NA & NA & NA \\
\cline { 2 - 6 } & Ref-OFDM & -27.1217 & -41.6051 & NA & NA & NA \\
\cline { 2 - 6 } & FBMC & -31.5356 & -45.7056 & NA & NA & NA \\
\hline
\end{tabular}

existing OFDM based LDACS is $-4.7 \mathrm{~dB}$ compared to $-5.5 \mathrm{~dB}$ in case single-band transmission for a given center frequency. As expected, it is much higher than $-41.5 \mathrm{~dB}$ interference at DME due to the proposed Ref-OFDM based LDACS.

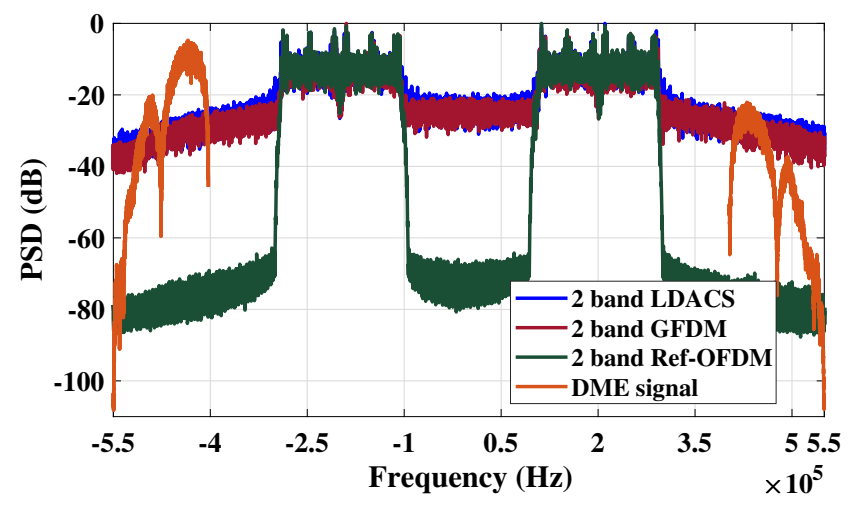

Fig. 15: The PSD comparison for 2-band transmission for ENR channel with bandwidth of $186 \mathrm{KHz}$.

For this case, we compared the BER performance of existing and proposed Ref-OFDM based LDACS for three different channel conditions. As shown in Figure 16, the performance of the Ref-OFDM based LDACS is significantly better than existing LDACS. Poor BER performance for narrow transmission bandwidth of $186 \mathrm{KHz}$ confirms the non-feasibility of existing LDACS for multi-band deployment.

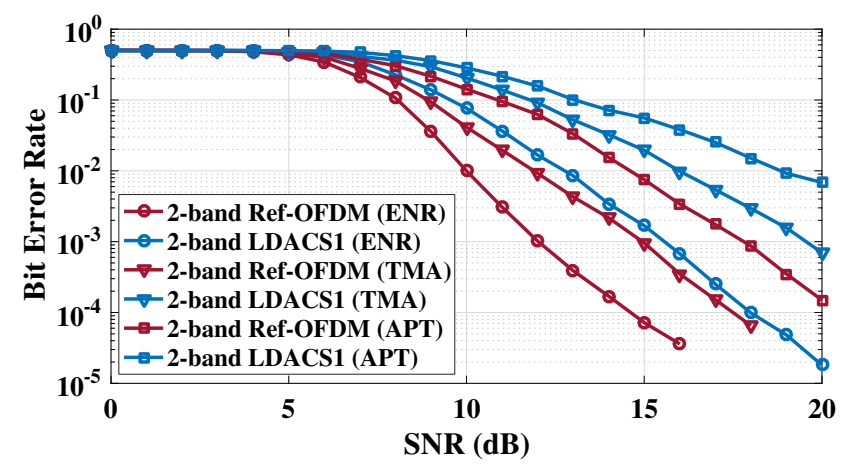

Fig. 16: The BER comparison of Ref-OFDM and OFDM based LDACS for three channels in presence of DME interference for 2-band transmission with $186 \mathrm{KHz}$ bandwidth. 


\section{Multi-user Transmission}

Next, we consider the scenario where two users share the frequency band where the transmission bandwidth of one user is $342 \mathrm{KHz}$, and another user is $186 \mathrm{KHz}$. The center frequencies are the same as that of 2-band transmission considered before. The corresponding PSD plots shown in Figure 15 indicates very high interference to the DME signal from existing LDACS. For instance, the DME interference due to existing LDACS is $4 \mathrm{~dB}$ compared to $-40 \mathrm{~dB}$ due to Ref-OFDM based LDACS.

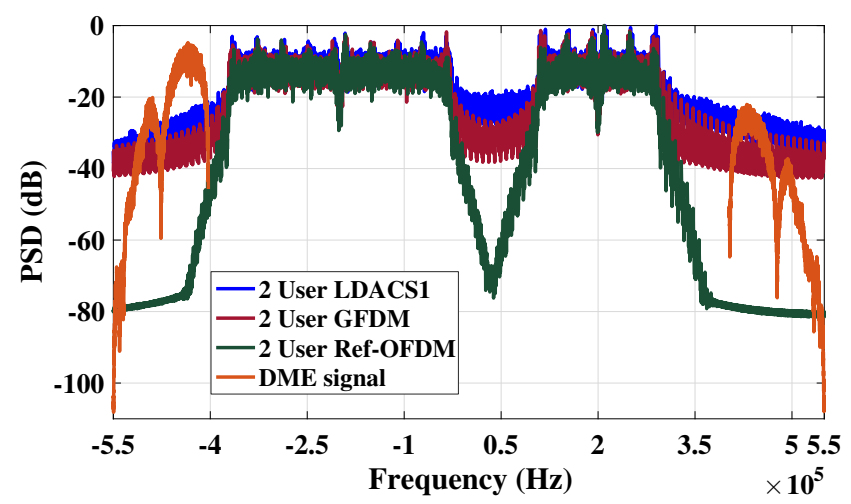

Fig. 17: The PSD comparison for 2-user transmission for ENR channel with bandwidth of $186 \mathrm{KHz}$ and $342 \mathrm{KHz}$.

For the above scenario, Figure 18 shows the BER performance of 2-user case for all three channel scenarios. It can be observed that the proposed Ref-OFDM based LDACS is significantly better than existing LDACS. These results also confirm the feasibility of multi-user transmission using Ref-OFDM based LDACS.

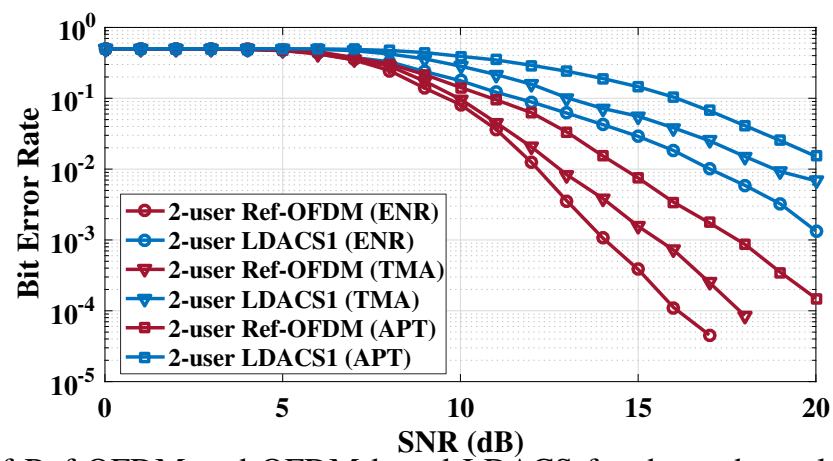

Fig. 18: The BER comparison of Ref-OFDM and OFDM based LDACS for three channels in presence of DME interference for 2-user transmission with $186 \mathrm{KHz}$ and $342 \mathrm{KHz}$ bandwidth.

\section{Complexity Comparison}

In this section, the complexity comparison of various waveforms regarding the number of real multiplications for different numbers of sub-carriers is done. Here, we consider $K$-band transmissions in non-continuous bands where $K \in\{2,4\}$. For such transmissions, Ref-OFDM uses a single reconfigurable filter capable of offering 16-band response. We also consider OFDM with conventional filter design and referred to as filtered-OFDM (F-OFDM). As shown in Figure 19, as $K$ increases, the complexity of F-OFDM increases while that of Ref-OFDM remains the same. The complexity of GFDM and FBMC with polyphase filter 
implementation for single band transmission is much higher than 16-band Ref-OFDM waveform. Also, the complexity of RefOFDM waveform is close to that of OFDM for 128 sub-carrier case making the proposed protocol and waveform a good alternative to next generation LDACS.

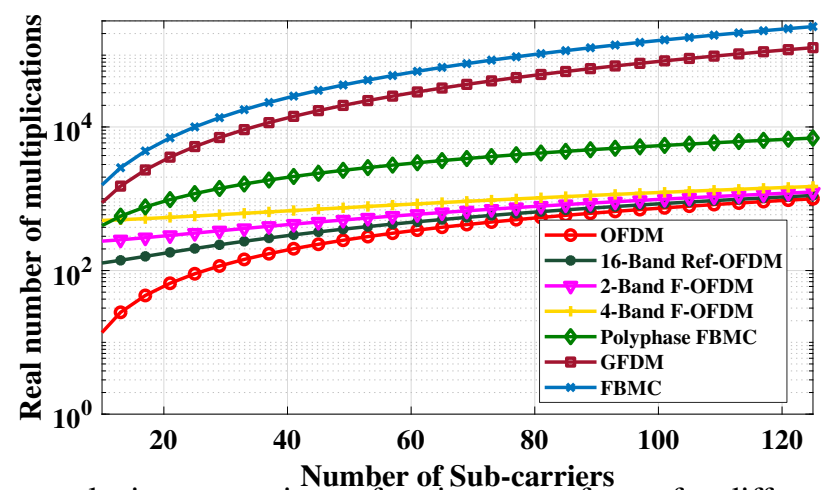

Fig. 19: Computational complexity comparison of various waveforms for different number of sub-carriers.

We have verified the throughput and effect of ADC quantization on the PSD of the Ref-OFDM in [4]. Also, the simulation results are verified using USRP based experimental results in [2]. In addition, the proposed Ref-OFDM based LDACS offers additional benefits. It provides flexibility via reconfigurable filters to enable transmission in non-contiguous bands, and lower OOB emission allows multiple transceivers share the vacant spectrum. Such sharing enables low data rate services which in turn can potentially eliminate the need for LDACS2. The proposed architecture can be efficiently realized on the FPGA via a dynamic partial reconfiguration approach. For instance, in the case of narrowband or single user transmission, the Ref-OFDM can switch to OFDM by bypassing the filter thereby saving the dynamic power. The dynamic partial reconfiguration can also be exploited to change between the single band and multi-band transmissions. For instance, in case of single band transmission, the DFT block can be reconfigured with the adder block. Such flexibility is difficult in case of OFDM, FBMC and GFDM based LDACS. Similar to OFDM, Ref-OFDM can be extended to a multi-antenna system, unlike FBMC based LDACS.

\section{CONCLUSION}

In this paper, we proposed a reconfigurable LDACS protocol with the frame structure that can adapt to different bandwidths and a reconfigurable filtered OFDM (Ref-OFDM) waveform using low complexity linear phase multi-band digital filter. The proposed protocol and Ref-OFDM enable transceivers to dynamically adapt the transmission bandwidth over a wide range to meet the desired quality of service, and high out-of-band attenuation allows wider transmission bandwidth as well as multiple narrowband transmissions. Simulation results show significant improvement over the BER and at least $32 \mathrm{~dB}$ lower interference to incumbent $L$-band users than existing LDACS. Furthermore, proposed work allows multi-band and multi-user transmission with adaptable bandwidth due to the proposed reconfigurable filter. Such transmission is not feasible in existing OFDM based LDACS due to significant interference to incumbent $L$-band users. Also, the computational complexity of Ref-OFDM is lower than other waveforms except OFDM making the proposed work an attractive solution for next-generation LDACS. Future works include an extension of the proposed work for air-to-air communication data link of the FCI system. 


\section{ACKNOWLEDGEMENT}

This work is supported by the Visvesvaraya Ph.D. fellowship and DST Inspire Fellowship granted by the Govt. of India.

\section{REFERENCES}

[1] Agrawal N., "New Reconfigurable L-Band Digital Aeronautical Communication System "http://arxiv.org/abs/1809.06033blue [blue] http://arxiv.org/abs/1809.06033.

[2] Agrawal N., Joshi H., Darak S. J., and Bader F., "USRP Testbed and Performance Analysis of New Reconfigurable LDACS In Presence of DME Interference," 2019 Integrated Communications, Navigation, Surveillance Conference (ICNS), Westin Washington Dulles Airport, Herndon, VA. (Submitted)

[3] Agrawal N., Darak S. J., and Bader F., "Reconfigurable filtered OFDM waveform for next generation air-to-ground communications," IEEE/AIAA 36th Digital Avionics Systems Conference (DASC), pp. 1-7, St. Petersburg, USA, Sept. 2017.

[4] Agrawal N., and Darak S. J. "Performance Analysis of Reconfigurable Filtered OFDM for LDACS"2019 11th International Conference on Communication Systems Networks (COMSNETS), Bengaluru.

[5] Ambede A., Prasad V. A., and Madhukumar A. S., "Design of a low complexity channel filter satisfying LDACS1 spectral mask specifications for air-to-ground communication," Integrated Communications Navigation and Surveillance (ICNS), pp. 1-7, Herndon, USA, Apr. 2016.

[6] Ambede A., Shreejith S., Prasad V. A., and Fahmy S. A., "Design and Realization of Variable Digital Filters for Software Defined Radio Channelizers using Improved Coefficient Decimation Method," in IEEE Transactions on CAS-II, vol. 62, no. 1, pp. 59-63, Jan. 2016.

[7] Brandes S., Epple U., Gligorevic S., Schnell M., Haindl B., and Sajatovic M., "Physical layer specification of the L-band Digital Aeronautical Communications System (L-DACS1), "IEEE Integrated Communications, Navigation and Surveillance Conference, pp. 1-12, Arlington, USA, May. 2009.

[8] B-VHF project, “Software Implementation of Broadband VHF channel models,"D-17, May 2006.

[9] B-VHF project, “Expected B-AMC system performance,"D-5, May 2006.

[10] Brandes S., Epple U., and Schnell M.. "Compensation of the impact of interference mitigation by pulse blanking in OFDM systems." IEEE Global Telecommunications Conference, pp. 1-6, Honolulu, USA, Dec. 2009.

[11] Cheng X., He Y., Ge B., and He C., "A Filtered OFDM Using FIR Filter Based on Window Function Method," 83rd IEEE Vehicular Technology Conference (VTC Spring), pp. 1-5, Nanjing, China, May. 2016.

[12] Depoorter D. G., and Kellerer W., "Designing the Air-Ground Data Links for Future Air Traffic Control Communications," in IEEE Transactions on Aerospace and Electronic Systems.

[13] Dhabu S., Prasad V. A., and Madhukumar A. S., "Low complexity fast filter bank-based channelization in L-DACS1 for aeronautical communications," IEEE 13th International New Circuits and Systems Conference (NEWCAS), pp. 1-4 ,Grenoble, France, Jun. 2015.

[14] Epple U., Hoffmann F., and Schnell M., "Modeling DME interference impact on LDACS1," IEEE Integrated Communications, Navigation and Surveillance Conference, pp. 1-13, Herndon, USA, Apr. 2012.

[15] Epple U. and Schnell M., "Overview of legacy systems in L-band and its influence on the future aeronautical communication system LDACS1," in IEEE Aerospace and Electronic Systems Magazine, vol. 29, no. 2, pp. 31-37, Feb. 2014.

[16] Epple U. and Schnell M., "Overview of interference situation and mitigation techniques for LDACS1," IEEE/AIAA 30th Digital Avionics Systems Conference (DASC), pp. 1-12, Seattle, USA, Sept. 2011.

[17] Eurocontrol, FAA, “Communications Operating Concept and Requirements for the Future Radio System,”COCR V2.0.

[18] Franzen N., Alexander A., and Schnell M., "L-DACS1 physical layer laboratory demonstrator." IEEE Integrated Communications Navigation and Surveillance Conference pp. 1-11, Herndon, USA, May 2010.

[19] Garg S., Agrawal N., Darak S. J., and Sikka P., "Spectral Coexistence of Candidate Waveforms and DME in Air-to-Ground Communications: Analysis via Hardware Software Co-Design on Zynq SoC, ”IEEE/AIAA 36th Digital Avionics Systems Conference, pp. 1-6, St. Petersburg, USA, Sept. 2017.

[20] Garg S., and Darak S. J., "FPGA Implementation of High Speed Reconfigurable Filter Bank for Multi-standard Wireless Communication Receivers, ” 20th International Symposium on VLSI Design and Test, pp. 1-5, Guwahati, India, May 2016.

[21] Gligorevic S., Epple U., and Schnell M. “The LDACS1 physical layer design,” INTECH Open Access Publisher, 2011. 
[22] Haider A., "Comparison of proposals for the Future Aeronautical Communication System LDACS, " Master Thesis, ILMENAU University of Technology and Institute of Communication and Navigation German Aerospace Center, 2012.

[23] Jamal H., and Matolak D. W., "FBMC and LDACS Performance for Future Air to Ground Communication Systems," in IEEE Transactions on Vehicular Technology, vol. 66, no. 6, pp. 5043-5055, June 2017.

[24] Kim E., "Improving DME Performance for APNT Using Alternative Pulse and Multipath Mitigation,” in IEEE Transactions on Aerospace and Electronic Systems, vol. 53, no. 2, pp. 877-887, April 2017.

[25] L-DACS2 project, “L-DACS2 System Definition Proposal,”D-2, Issue 1.0, May 2009.

[26] Ma L., and Zhang C., "5G waveforms design for aeronautical communications," IEEE/AIAA 35th Digital Avionics Systems Conference (DASC), pp. 1-7, Sacramento, CA, Sept. 2016.

[27] Mahesh R., and Prasad V. A., "Coefficient decimation approach for realizing reconfigurable finite impulse response filters," IEEE Intl. Symp. On Circuits and Systems, (ISCAS), pp. 81-84, Washington, USA, May 2008.

[28] Michailow N., Matth M., Gaspar I. S., Caldevilla A. N., Mendes L. L., Festag A., and Fettweis G., "Generalized Frequency Division Multiplexing for 5th Generation Cellular Networks," in IEEE Transactions on Communications, vol. 62, no. 9, pp. 3045-3061, Sept. 2014.

[29] Neji N., De Lacerda R., Azoulay A., Letertre T., and Outtier O., "Survey on the Future Aeronautical Communication System and Its Development for Continental Communications," in IEEE Transactions on Vehicular Technology, vol. 62, no. 1, pp. 182-191, Jan. 2013.

[30] Pham T. H., Prasad V. A., and Madhukumar A. S., "A Hardware-Efficient Synchronization in L-DACS1 for Aeronautical Communications," in IEEE Transactions on Very Large Scale Integration (VLSI) Systems, vol. 26, no. 5, pp. 924-932, May 2018.

[31] Pham T. H., Prasad V. A., and Madhukumar A. S., "An efficient data aided synchronization in L-DACS1 for aeronautical communications," Proc. ACM Int. Conf. Data Mining, Commun. Inf. Technol. (DMCIT), pp. 15, Phuket, Thailand, May 2017.

[32] Proakis J., and Salehi M., Digital Communications, McGraw-Hill, 2008.

[33] Sajatovic M., Haindl B., Ehammer M., Grupl T., Schnell M., Epple U., and Brandes S., "L-DACS1 System Definition Proposal: Deliverable D2,” in Technical Report, Eurocontrol, no. 1.0, Feb 2009.

[34] Schneckenburger N., Franzen N., Gligorevic S., and Schnell M., "L-band compatibility of LDACS1," IEEE/AIAA 30th Digital Avionics Systems Conference (DASC), pp. 1-11, Seattle, USA, Oct. 2011.

[35] Schnell M., Epple U., Shutin D., and Schneckenburger N., "LDACS: future aeronautical communications for air-traffic management," in IEEE Communications Magazine, vol. 52, no. 5, pp. 104-110, May. 2014.

[36] Schnell M., Franzen N., and Gligorevic S., "L-DACS1 laboratory demonstrator development and compatibility measurement set-up," IEEE/AIAA 29th Digital Avionics Systems Conference (DASC), pp. 1-11, Salt Lake City, USA, Oct. 2010.

[37] SESAR Joint Undertaking, "NextGen SESAR State of Harmonisation," in Federal Aviation Administration, June. 2016.

[38] Shreejith S., Ambede A., Prasad V. A., and Fahmy S. A., "A power and time efficient radio architecture for LDACS1 air-to-ground communication," IEEE/AIAA 35th Digital Avionics Systems Conference (DASC), pp. 1-6, Sacramento, USA, Sept. 2016.

[39] Shreejith S., Mathew L. K., Prasad V. A., and Fahmy S. A., "Efficient spectrum sensing for aeronautical LDACS using low-power correlators", in IEEE Transactions on Very Large Scale Integrated Systems, vol. 26, no. 6, pp. 1183-1191, June 2018.

[40] Solla T., Vainio O., "Comparison of Programmable FIR Filter Architectures for Low Power," Proc. of 28 ${ }^{\text {th }}$ European Solid-State Circuits Conference, pp. 759-762, Italy, Sep. 2002.

[41] STATFOR, the EUROCONTROL Statistics and Forecast Service, "Challenges of Growth, Task 4: European Air Traffic in 2035," EUROCONTROL, June. 2013.

[42] Stoyanov G., and Kawamata M., "Variable Digital Filters," in J. Signal Processing, vol. 1, no. 4, pp. 275-289, July 1997.

[43] Viholainen A., Bellanger M., and Huchard M., "Prototype filter and structure optimization: Deliverable D5.1.” Technical report PHYDYAS, 2009. 


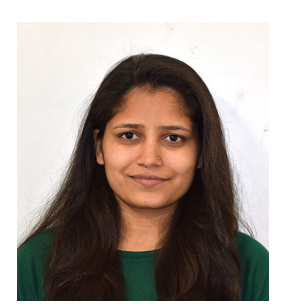

Niharika Agrawal received her B.Tech. degree in Electronics and Communication Engineering (ECE) from Poornima Institute of Information Technology, India in 2013 and the M.Tech degree from LNM Institute of Information Technology, India in 2015. She is currently pursuing the $\mathrm{PhD}$ degree with the Algorithms to Architecture laboratory at Indraprastha Institute of Information Technology, Delhi. Her current research interests are waveform design for Air to Ground communication, hardware implementation of end to end transceivers.

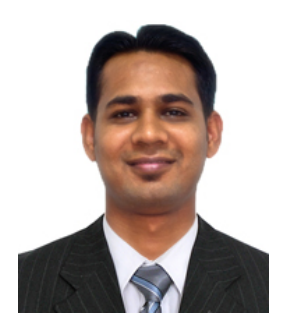

Sumit J. Darak received his Bachelor of Engineering (B.E.) degree in Electronics and Telecommunications Engineering from Pune University, India in 2007, and PhD degree from the School of Computer Engineering, Nanyang Technological University (NTU), Singapore in 2013. He is currently an Assistant Professor at Indraprastha Institute of Information Technology, Delhi, India. Prior to that, he was working as Assistant System Engineer in Tata Consultancy Services (TCS), Pune, India from September 2007 to December 2008. From August 2011 to November 2011, he was visiting research student at Massey University, Auckland, New Zealand. From August 2012 to January 2013, he worked as an intern at EADS Innovation Works (Airbus), Singapore. From March 2013 to November 2014, he was pursuing postdoctoral research at the CominLabs Excellence Center, Universit Europenne de Bretagne (UEB) and Suplec, Rennes, France for the project GREAT: Green Cognitive Radio for Energy-Aware Wireless Communication Technologies Evolution. Dr. Sumit has been awarded India Governments DST Inspire Faculty Award which is a prestigious award for young researchers under 32 years age. His research interests include design and implementation of multistandard wireless communication receivers as well as application of machine learning algorithms and decision making policies for various wireless communication applications.

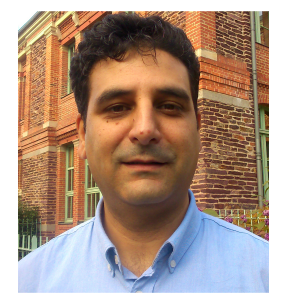

Faouzi Bader received the Ph.D. degree (with Honours) in telecommunications from Universidad Politecnica de Madrid (UPM), Madrid, Spain, in 2002. He joined the Centre Technologic de Telecomunicacions de Catalunya (CTTC), BarcelonaSpain, as Associate Researcher, in 2002, and was nominated in 2006 as a Senior Research Associate at same institution. Since June 2013, he is as Associate Professor at Ecole Superieure dElectricite-Supelec in Rennes-France, and since 2017 as honorary Adjunct Professor at University of Technology, Sydney Australia . His research activities mainly focus on IMT-Advanced systems, advanced multicarrier waveforms, and frequency allocation techniques in relay cognitive environments. He has published over 120 papers in peer reviewed journals and international conferences, more than 13 book chapters, and edited 4 books. He served as Technical Program Committee member in major IEEE ComSoc and VTS conferences, as associate editor in Emerging Telecommunications Technologies-ETT Journal (2013-2016), and as guest editor at EURASIP Journal on Advances in Signal Processing (JASP). 


\section{LIST OF FIGURES}

Various communication links in the future communication infrastructure (FCI) system. . . . . . . . . . . 2

Brief history about the evolution of $\mathrm{A} 2 \mathrm{GC}$ system. . . . . . . . . . . . . . . . . . . . 2

4 Proposed frame structure for the revised LDACS protocol depicting the data, pilot and synchronization symbol patterns and locations for $732 \mathrm{KHz}$ transmission bandwidth. . . . . . . . . . . . . . . . . . .

Proposed frame structure for the revised LDACS protocol depicting the data, pilot and synchronization symbol patterns and locations for the transmission bandwidth of (a) $498 \mathrm{KHz}$ and (b) $186 \mathrm{KHz}$. . . . . . . . . . . 7

(a) Single user, and (b) Multi-user LDACS deployment scenarios for a given DME interference threshold. . . . . .

Reconfigurable filter design using CDM and MCDM. (a) Prototype baseband bandpass filter with $\omega_{c}=0.12 \pi$ (b) Baseband bandpass filter responses with the bandwidth $0.24 \pi$ and $0.72 \pi$ obtained using the CDM approach with $D=2$ and $D=6$, respectively, (c) Baseband bandpass filter responses with the bandwidth $0.76 \pi$ obtained using the MCDM with $D=2$

Variable baseband bandpass frequency responses obtained using fixed-coefficient baseband bandpass prototype filter with $\omega_{c}=0.12 \pi$ and, (a) CDM , and (b) MCDM approach. . . . . . . . . . . . . . . . . . . 10

12 The PSD comparison of various waveforms for ENR channel and two different transmission bandwidths, (a) 732KHz, and (b) $498 \mathrm{KHz} \ldots \ldots \ldots \ldots \ldots \ldots$

13 The BER comparison of various waveforms for two different transmission bandwidths, (a) $732 \mathrm{KHz}$, and (b) $498 \mathrm{KHz}$

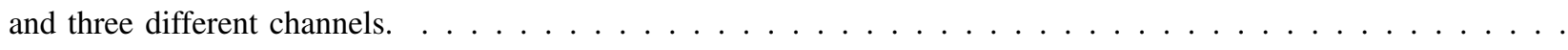

14 The BER comparison of Ref-OFDM and OFDM based LDACS in presence of DME interference for ENR channel with $342 \mathrm{KHz}$ transmission bandwidth and three different center frequencies. . . . . . . . . . . . . . . . 18

15 The PSD comparison for 2-band transmission for ENR channel with bandwidth of $186 \mathrm{KHz}$. . . . . . . . . . .

16 The BER comparison of Ref-OFDM and OFDM based LDACS for three channels in presence of DME interference for 2-band transmission with $186 \mathrm{KHz}$ bandwidth. . . . . . . . . . . . . . . . . . . . . . 19

17 The PSD comparison for 2-user transmission for ENR channel with bandwidth of $186 \mathrm{KHz}$ and $342 \mathrm{KHz}$. . . .

18 The BER comparison of Ref-OFDM and OFDM based LDACS for three channels in presence of DME interference for 2-user transmission with $186 \mathrm{KHz}$ and $342 \mathrm{KHz}$ bandwidth. . . . . . . . . . . . . . . . . . . . 20 